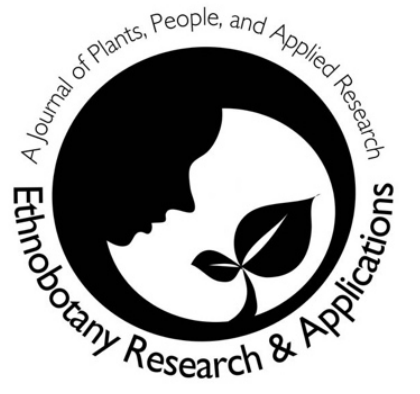

\title{
Ethnobotany of the genus Elatostema J.R. Forster \& G. Forster (Urticaceae)
}

Ashutosh Kumar Upadhyay, Rajib Gogoi and Prashanta Kumar Mitra

\section{Reviews and Mini reviews}

\begin{abstract}
Background: Wild edible plants (WEP's) are used in various traditional systems all over the globe. WEP's are used as food, medicine, ornamental, forage and material purposes. Elatostema is one such genus that is used by many indigenous communities in Africa, Asia and Oceania. The objectives of this study are to provide ethnobotanical information about the non-stinging nettle genus Elatostema, to identify indigenous communities that use these species and to examine leaf area variation in species used by ethnic communities.
\end{abstract}

Methods: The data sets were collected from various online sources such as Research gate, Google Scholar and Academia.edu; print sources such as published articles in ethnobotanical journals and herbarium sources from various herbaria. The datasets were then segregated into four types (Consumption, medicinal, Forage and other) based on the recorded usage by indigenous communities all over the globe. We analyzed leaf variation with type of usage using density plots, Shapiro-Wilks test, Kruskal-Wallis test and Principal Component Analysis (PCA). The Use-Value (UV) of all species was also calculated using the number of recorded usage and their citations within the genus.

Results: This study documented 40 Elatostema species with recorded ethnobotanical usage. We record 30 indigenous communities that use species of Elatostema in their daily lives. After performing all the analysis (density plots, Shapiro-Wilks test, Kruskal-Wallis test and Principal Component Analysis) we found out that there is no significant variation in leaf area with the type of use. Elatostema platyphyllum Wedd. and Elatostema sessile J.R. Forster \& G. Forster were found to have low values $(U V=0.18$ and $U V=0.23)$ because of high recorded usage and citations.
Conclusion: This study illustrates diversity of Elatostema species used as food, medicine, forage and ornamental or material purposes by various indigenous communities in the world. The study disproves our hypothesis that the usage of an Elatostema species for a specific purpose (food, medicine, fodder, others) is related to leaf size, i.e., bigger the size of the leaves, more the chances of it to be used as food or forage. Results from our analyses shows that there is no variation in leaf area with the type of use. The documented ethnobotanical records of Elatostema species along with our personal observations in the field provided in this study would help in elucidating the importance of this genus as one of the main leafy wild edible plant for human consumption and will further promote applied research in this group.

Key words: Elatostema, Urticaceae, ethnobotany, Indigenous communities, Analysis

\section{Correspondence}

Ashutosh Kumar Upadhyay ${ }^{1^{*}, 2}$, Rajib Gogoi ${ }^{1}$ \& Prashanta Kumar Mitra ${ }^{3}$

${ }^{1}$ Botanical Survey of India, Sikkim Himalayan Regional Centre, Gangtok, Sikkim-737103

${ }^{2}$ Mansarovar Global University, Billkisganj, Sehore, Madhya Pradesh-466001

${ }^{3}$ Department of Botany, University of Kalyani, Nadia, West Bengal-741235

"Corresponding Author:

ashutoshpdh196@gmail.com

Ethnobotany Research \& Applications 21:07 (2021) 


\section{Background}

Human beings have been cultivating, growing and improving plants for their benefit and they collect certain species for their use. This relationship between human beings and plants is what forms the base for ethnobotany (Jain \& Dam 1979). Plant species collected from the wild have become a major source of nutrition and medicine for many indigenous people (Heywood 2011). Their life is dependent on forest for access to natural plant wealth which provides them food and other materials required for their sustenance (Sundriyal et al. 1998; Arora \& Pandey 1996; Albuquerque \& Hanazaki 2009). Moreover, the usage of wild plants is an integral part of strong traditional and cultural systems which has been developed over generations (Sundriyal et al. 1998). Documenting indigenous knowledge of plants and their ethnobiological value is significant to study human plant relationships, conservation and utilization of biological resources (Pradhan \& Badola 2008; Pieroni et al. 2011). The wild edible plants especially the ones used as leafy vegetables makes significant contribution in generation of income (Angami et al. 2006). The wild edible plants are considered as the most important part of the Nontimber Forest Products (NTFPs) and they play a major role in elevating the socio-economic status of tribal and ethnic communities (Maikhuri et al. 2004). In many studies Elatostema species have been recorded to be used as a non-timber forest product by various indigenous groups (Murtem \& Chaudhry 2016a; Angami et al. 2006; Muthu \& Rimo 2018; Srivastava \& Nyishi 2010; Shrestha 2013; Konsam et al. 2016a).

While exploring wild plant species with potential for human consumption it is important to consider recorded ethnobotanical uses, their geographical occurrence and their morphology. These data help in asking relevant questions such as how many species are used by human beings and for what purposes, why only a specific species is used in an area and is the usage dependent on the morphology of the species (Miller et al. 2015).

Genus Elatostema J.R. Forster \& G. Forster, of the family Urticaceae are usually herbs, sub- shrubs and rarely shrubs. It consists of about 500 species distributed in the tropical and Sub-tropical Africa, Asia, Oceania but is absent in the Neotropics (Qi et al. 2003; Wei et al. 2011; Tseng et al. 2019). The International Plant Name Index search shows 995 names for Elatostema complex which includes sectional and serial names. These plants grow luxuriantly in the tropical, sub-tropical and subtemperate forests having an elevation between 500 $2500 \mathrm{~m}$ (Tseng et al. 2019). This genus is found to be most diverse on limestone karsts of South East Asia (Qi et al. 2003). The delimitation of this genus along with Elatostematoides C.B. Rob., Procris Comm. ex Juss. and Pellionia Gaudich. has been problematic since its inception (Hadiah et al. 2003; Hadiah \& Conn 2009; Tseng et al. 2019; Wu et al. 2013).

Elatostema species are believed to be of a meagre economical usage which are used in combination with other plants for treating cough, flu, bowel movements, itching, etc. (Wei et al. 2011). Leaves of species like Elatostema platyphyllum Wedd., E. sessile J.R. Forst. \& G. Forst. are consumed by many indigenous communities such as Nyishi, Adi, Mao, etc. in North-East India (Tshering et al. 2018; Murtem \& Chaudhry 2016b; Pfoze et al. 2012; Sigdel et al. 2013; Yumnam et al. 2011; Ronald et al. 2019; Angami et al. 2006; Muthu \& Rimo 2018; Srivastava \& Nyishi 2010). The information regarding the number of species used by people from indigenous communities and their type of usage is scanty and scattered across various literature. This has led to few applied studies in this genus which focuses on disease management, anti-bacterial activity, nutraceutical properties and trace elements (Konsam et al. 2016b; Mariani et al. 2016; Reza et al. 2018; Santos et al. 2018; Hui 2019). In our study we provide a compilation of documented ethnobotanical records along with our personal observations in the field which we believe would help in elucidating the importance of this genus as a major wild edible plant for human consumption and will further promote applied research in this genus.

The objectives of this study are to (1) provide a global ethnobotanical data of the non-stinging nettle genus Elatostema, (2) to identify the indigenous communities across the globe that use these species for various purposes and (3) to assess if there is any significant difference in leaf area with type of usage (Human consumption, Medicinal use, Forage and Other use), i.e., if the species with bigger leaf areas were preferred more as food or fodder or species with smaller leaves were used more as medicine, etc.

\section{Materials and Methods}

\section{Data collection}

We collected data on usage of different plant parts by indigenous communities along with data on geographic distribution of the Elatostema species from herbarium specimens housed at CAL, ARUN, ASSAM, DD, BSHC, K, BM, NY, and P (Herbarium codes following Thiers 2016), (Table 1). 
Table 1. Indigenous communities recorded in the study

\begin{tabular}{|l|l|l|l|}
\hline \multicolumn{1}{|c|}{$\begin{array}{c}\text { Indigenous } \\
\text { communities }\end{array}$} & \multicolumn{1}{c|}{ Region } & \multicolumn{1}{c|}{$\begin{array}{c}\text { Indigenous } \\
\text { communities }\end{array}$} & \multicolumn{1}{c|}{ Country } \\
\hline Jaintia & Meghalaya & Melpa & PAPUA NEW GUINEA \\
\hline Monpa & Arunachal Pradesh & Kaili Inde & INDONESIA \\
\hline Nyishi & Arunachal Pradesh & Monpa & CHINA \\
\hline Adi & Arunachal Pradesh & Chagga & TANZANIA \\
\hline Mao & Manipur & Batak Toba & INDONESIA \\
\hline Koki & Manipur & Chakma & BANGLADESH \\
\hline Mizo & Mizoram & Han & CHINA \\
\hline Angami & Nagaland & Lua & THAILAND \\
\hline $\begin{array}{l}\text { Karbi and Munda } \\
\text { communities }\end{array}$ & Assam & Hani & CHINA \\
\hline Apatani & Arunachal Pradesh & Siwai & INDONESIA \\
\hline Limboo & Sikkim & Buin & INDONESIA \\
\hline Tangkhul & Manipur & Ndumba & PAPUA NEW GUINEA \\
\hline Cholanaikan & Kerala & Samawa community & INDONESIA \\
\hline Miji & Arunachal Pradesh & Batak Simalungun & INDONESIA \\
\hline Galo & Arunachal Pradesh & Kalam & PAPUA NEW GUINEA \\
\hline
\end{tabular}

We also noted the relevant data viz. location, date, collection number, collector, etc. Data from the field trips from December 2018 to March 2020 in North East India (Arunachal Pradesh, Meghalaya and Sikkim) were collected using informal interviews and a simple non-compulsory, open-ended questionnaire wherein the informants did not have to answer if they felt uncomfortable. The elders of different indigenous communities between the age groups of 45 to 65 years (village heads, foresters etc.) who frequently visited the forests were asked questions about the usage of Elatostema species in their daily lives. Local tribal markets were also visited to record plant usage. The voucher number of species collected during these tours are provided in Table 2. A literature review was undertaken to investigate, and document recorded uses of Elatostema species. We used various scientific literature sources such as Research Gate, Google scholar and Academia.edu and used keywords such as "Elatostema", "Ethnobotany of Elatostema", "uses of Elatostema" for searching relevant data on the usage of Elatostema species in the world. We reviewed (1) ethnobotanical studies carried out in the regions where Elatostema is recorded to occur and (2) studies on the usage of wild edible plants by various indigenous communities across the world. We consulted online ethnobotanical database Plants for future and scientific databases such as JSTOR, Pubmed, Plants of the World online, Global Biodiversity Information Facility, International Plant Names Index and The Plant list for information on Elatostema species. A map was prepared based on recorded ethnobotanical data of collected and documented species from various parts of the world (Figure 1).

\section{Leaf area measurements}

We examined herbarium specimens housed at Central National Herbarium (CAL), Kolkata that were collected from the locality from which the ethnobotanical data were recorded. Linear measurements were used for calculating leaf area of Elatostema species (Bhat \& Chanda 2003). Herbarium specimens were also consulted online $(K$, $\mathrm{BM}, \mathrm{NY}$, and $\mathrm{P}$ ) for specimens that were not found at CAL (Herbarium codes following Thiers 2016). The protologues of species with documented ethnobotanical usage were checked online using Biodiversity Heritage Library (BHL) for leaf dimensions when neither digital images nor physical specimens could be found.

In our study we recorded 40 species (Table 2) with documented ethnobotanical usage. Of these we could collect leaf area of 27 taxa that were identified up to the rank of species in various literary sources,13 taxa (not identified up to the rank of species in literary sources) were not used in leaf area measurements. We investigated three herbarium specimens per species and noted the range in leaf dimensions from 5-6 leaves per species and the average of these ranges were then used to calculate the area. We calculated leaf area using direct methods (linear measurement from leaves) and graph paper method. 


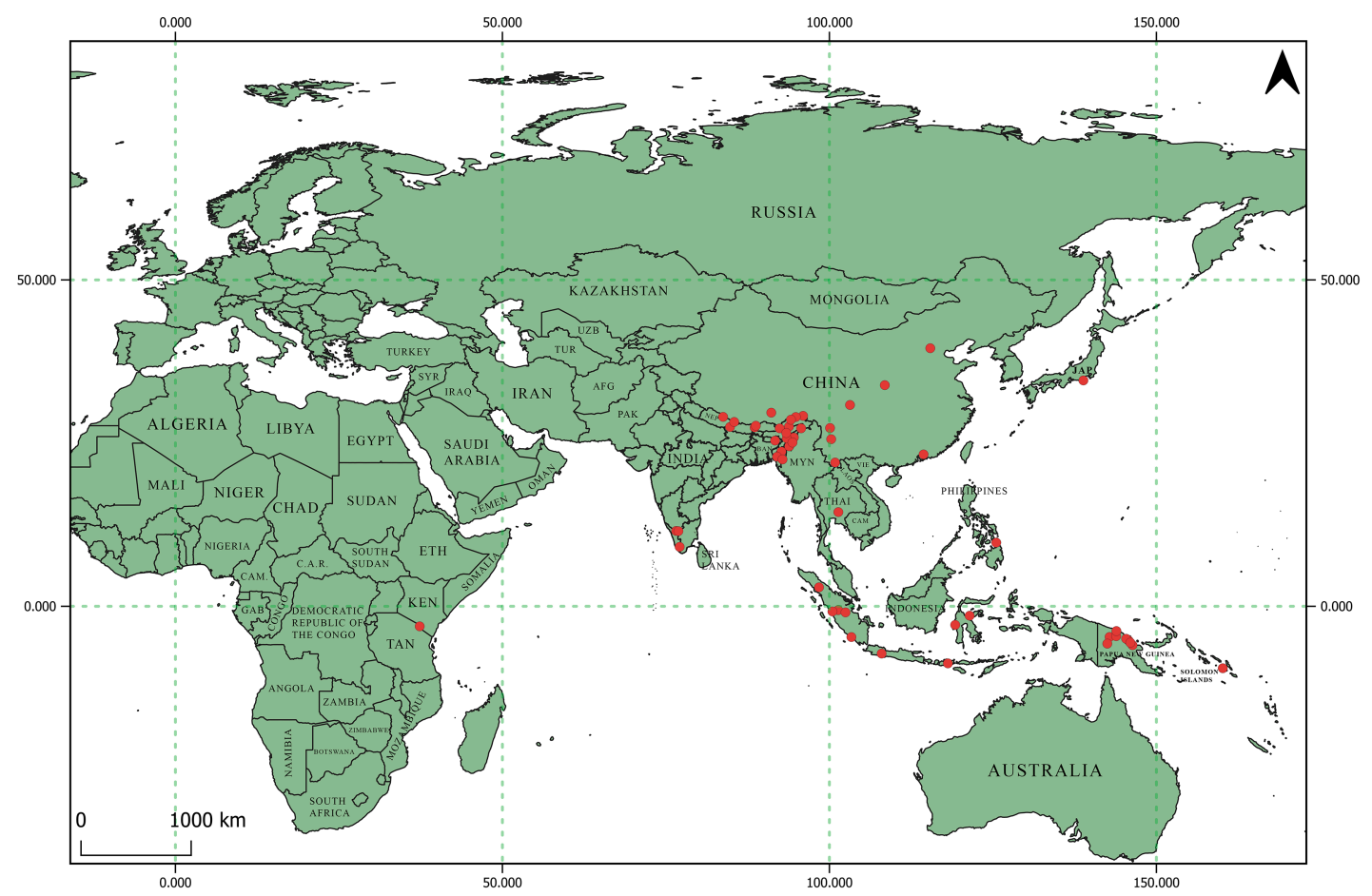

Figure 1. Geographic locations of species documented in this study. Collection sites determined by herbarium specimens and literature survey.

\section{Data analysis}

Ethnobotanical data were categorized as plant parts used, usage as food, medicinal uses, forage and others (ornamental, material uses etc.) The food category comprises of species that are used for human consumption; the medicinal category comprises of species used in varied forms for healing and therapeutical effects; the forage category comprises of species collected from the wild especially for feeding domesticated animals; finally, the others category comprises of species used for ornamentation, counter-magic and material purposes such as making ropes.

To assess if there is any significant difference in leaf area regarding type of use (Human consumption, Medicinal use, Forage, Other use) we used $\mathrm{HO}$ (null hypothesis) and $\mathrm{H} 1$ (our hypothesis)

$\mathrm{HO}$ : There is no significant difference in leaf area regarding type of use.

$H 1$ : There is a significant difference in leaf area regarding type of use.

Our data set had two independent categorical variables (1. Use, 2. Status) and one dependent numerical variable (Leaf area). Variable use has four levels (Human-consumption, Medicinal-use, Forage, Other-use) and variable status has two levels (Recorded, Not-recorded). Groups in variable use
(Human-consumption, Medicinal-use, Forage, Other-use) are treated as main groups and groups in variable status are treated as subgroups of the main. First the data was visually analyzed. Shapiro-Wilks test was performed to test normality. As all the data were found to be non-parametric, Kruskal-Wallis rank sum test was done for analyzing significant difference. For better understanding of how all parameters correlate and the data distribution Principal Component Analysis (PCA) was done. Statistical analysis and making of plots were done using $\mathrm{R}$ studio3.0.

\section{Use-value (UV)}

Ethnobotanical studies seek to identify important plant species for a given culture. In order to make conclusions about plant-human knowledge across cultures we must be able to measure ethnobotanical knowledge in a uniform way (Zenderland et al. 2019). Use-value index is widely used for the purpose of quantifying the importance of useful plants (Phillips \& Gentry 1993). Formula given by Rossato et al. (1999) $\left(U V=\Sigma U_{i} /\right.$ ) is used for these studies. $U_{i}$ is total number of uses recorded for a species while, $n$ is total number of informants or frequency of citation. We used Use-Value Index across recorded categories (Food, Medicinal, Forage and Others) to find out the relation between number of recorded uses per species and their actual Use value in our datasets. 
Table 2. Documentation of Elatostema species with recorded Ethnobotanical usage

\begin{tabular}{|c|c|c|c|c|c|c|c|c|c|c|}
\hline Species & $\begin{array}{l}\text { Voucher } \\
\text { numbers }\end{array}$ & Region & $\begin{array}{l}\text { Indigenous } \\
\text { communities }\end{array}$ & $\begin{array}{l}\text { Local } \\
\text { names }\end{array}$ & $\begin{array}{l}\text { Parts } \\
\text { used }\end{array}$ & $\begin{array}{l}\text { Usage as } \\
\text { food }\end{array}$ & Medicinal Uses & $\begin{array}{l}\text { Used as } \\
\text { forage }\end{array}$ & $\begin{array}{l}\text { Others } \\
\text { (Ornamental/ } \\
\text { Material uses) }\end{array}$ & References \\
\hline $\begin{array}{l}\text { Elatostema } \\
\text { acuminatum } \\
\text { (Poir.) Brongn. }\end{array}$ & 71648 & CHINA & Han tribe & $\begin{array}{l}\text { Not } \\
\text { mentioned }\end{array}$ & $\begin{array}{l}\text { Leaves, } \\
\text { fruits }\end{array}$ & $\begin{array}{l}\text { Edible, used } \\
\text { as leafy } \\
\text { vegetable }\end{array}$ & Not mentioned & $\begin{array}{l}\text { Not } \\
\text { mentioned }\end{array}$ & Not mentioned & $\begin{array}{l}\text { Ghorbani et al. } \\
2012\end{array}$ \\
\hline $\begin{array}{l}\text { Elatostema } \\
\text { beccarii } \mathrm{H} . \\
\text { Schroet. }\end{array}$ & $\begin{array}{l}\text { Not } \\
\text { Collected }\end{array}$ & $\begin{array}{l}\text { PAPUA NEW } \\
\text { GUINEA }\end{array}$ & Melpa tribe & Kengana & Leaves & $\begin{array}{l}\text { Not } \\
\text { mentioned }\end{array}$ & Not mentioned & $\begin{array}{l}\text { Not } \\
\text { mentioned }\end{array}$ & Counter magic & Telban 1988 \\
\hline $\begin{array}{l}\text { Elatostema } \\
\text { cuneatum Wight }\end{array}$ & $\begin{array}{l}\text { Not } \\
\text { Collected }\end{array}$ & $\begin{array}{l}\text { INDIA } \\
\text { (Arunachal } \\
\text { Pradesh) }\end{array}$ & $\begin{array}{l}\text { Monpa } \\
\text { community }\end{array}$ & Chulukpa & Leaves & $\begin{array}{l}\text { Tender } \\
\text { Leaves } \\
\text { consumed } \\
\text { as vegetable } \\
\text { after proper } \\
\text { leaching }\end{array}$ & Not mentioned & $\begin{array}{l}\text { Not } \\
\text { mentioned }\end{array}$ & Not mentioned & $\begin{array}{l}\text { Plants for a } \\
\text { Future } \\
\text { (https://pfaf.org/); } \\
\text { Tsering et al. } \\
2017\end{array}$ \\
\hline $\begin{array}{l}\text { Elatostema } \\
\text { cuneiforme W.T. } \\
\text { Wang }\end{array}$ & $\begin{array}{l}\text { Not } \\
\text { Collected }\end{array}$ & CHINA & $\begin{array}{l}\text { Monpa } \\
\text { community }\end{array}$ & $\begin{array}{l}\text { Tsen-tsen- } \\
\text { pa }\end{array}$ & Stem & $\begin{array}{l}\text { Not } \\
\text { mentioned }\end{array}$ & Not mentioned & $\begin{array}{l}\text { Aerial } \\
\text { parts used } \\
\text { as forage }\end{array}$ & $\begin{array}{l}\text { Stem used as } \\
\text { rope or twine }\end{array}$ & Li et al. 2020 \\
\hline $\begin{array}{l}\text { Elatostema } \\
\text { dissectum Wedd. }\end{array}$ & 71643 & $\begin{array}{l}\text { INDIA } \\
\text { (Meghalaya, } \\
\text { Arunachal } \\
\text { Pradesh) }\end{array}$ & Jaintia tribe & Jhur Khlow & $\begin{array}{l}\text { Leaves, } \\
\text { Fruits }\end{array}$ & $\begin{array}{l}\text { Raw or } \\
\text { Young } \\
\text { Leaves } \\
\text { cooked }\end{array}$ & Not mentioned & $\begin{array}{l}\text { Not } \\
\text { mentioned }\end{array}$ & Not mentioned & $\begin{array}{l}\text { Plants for a } \\
\text { Future } \\
\text { (https://pfaf.org/); } \\
\text { Jain \& Dam 1979; } \\
\text { Jaiswal 2010 }\end{array}$ \\
\hline $\begin{array}{l}\text { Elatostema } \\
\text { ficoides Wedd. }\end{array}$ & $\begin{array}{l}\text { Not } \\
\text { Collected }\end{array}$ & INDIA(Sikkim) & Not mentioned & $\begin{array}{l}\text { Chiplu, } \\
\text { Chiplay }\end{array}$ & $\begin{array}{l}\text { Not } \\
\text { mentioned }\end{array}$ & $\begin{array}{l}\text { Not } \\
\text { mentioned }\end{array}$ & $\begin{array}{l}\text { Ethnoveterinary } \\
\text { medicine. } 20- \\
50 \text { gm plants } \\
\text { crushed and given } \\
\text { twice daily to } \\
\text { lifestock to treat } \\
\text { fever }\end{array}$ & $\begin{array}{l}\text { Not } \\
\text { mentioned }\end{array}$ & Not mentioned & $\begin{array}{l}\text { Bharti \& Sharma } \\
2012\end{array}$ \\
\hline $\begin{array}{l}\text { Elatostema } \\
\text { heyneanum } \\
\text { (Wedd.) Hallier f. }\end{array}$ & $\begin{array}{l}\text { Not } \\
\text { Collected }\end{array}$ & INDIA (Kerala) & $\begin{array}{l}\text { Cholanaikan } \\
\text { tribe }\end{array}$ & Elaven & Leaves & $\begin{array}{l}\text { Not } \\
\text { mentioned }\end{array}$ & $\begin{array}{l}\text { Leaf juice is used } \\
\text { for treating } \\
\text { immunodeficiency } \\
\text { in children and } \\
\text { various liver } \\
\text { diseases }\end{array}$ & $\begin{array}{l}\text { Not } \\
\text { mentioned }\end{array}$ & Not mentioned & $\begin{array}{l}\text { Vilash et al. 2016; } \\
\text { Kumar et al. } 2019\end{array}$ \\
\hline
\end{tabular}




\begin{tabular}{|c|c|c|c|c|c|c|c|c|c|c|}
\hline $\begin{array}{l}\text { Elatostema } \\
\text { hookerianum } \\
\text { Wedd. }\end{array}$ & 71638 & $\begin{array}{l}\text { CHINA } \\
\text { (Yunnan) }\end{array}$ & Not mentioned & $\begin{array}{l}\text { Kena, } \\
\text { Shilikangqui } \\
\text { ng }\end{array}$ & $\begin{array}{l}\text { Whole } \\
\text { plant }\end{array}$ & $\begin{array}{l}\text { Not } \\
\text { mentioned }\end{array}$ & Not mentioned & $\begin{array}{l}\text { Used as } \\
\text { fodder to } \\
\text { feed } \\
\text { Mithun } \\
\text { (Bos } \\
\text { frontalis) }\end{array}$ & Not mentioned & Geng et al. 2017 \\
\hline $\begin{array}{l}\text { Elatostema } \\
\text { integrifolium (D. } \\
\text { Don) Wedd. }\end{array}$ & 71647 & $\begin{array}{l}\text { NEPAL (N.Lipe } \\
\text { Jhar) }\end{array}$ & Not mentioned & $\begin{array}{l}\text { Not } \\
\text { mentioned }\end{array}$ & Root & $\begin{array}{l}\text { Not } \\
\text { mentioned }\end{array}$ & $\begin{array}{l}\text { Juice of Root } \\
\text { about } 4 \text { teaspoon } \\
\text { is given } 3 \text { times a } \\
\text { day in case of } \\
\text { fever }\end{array}$ & $\begin{array}{l}\text { Not } \\
\text { mentioned }\end{array}$ & Not mentioned & Manandhar 1993 \\
\hline $\begin{array}{l}\text { Elatostema } \\
\text { involucratum } \\
\text { Franch. \& Sav. }\end{array}$ & $\begin{array}{l}\text { Not } \\
\text { Collected }\end{array}$ & CHINA & Hani tribe & Luo bu, A bo & $\begin{array}{l}\text { Tender } \\
\text { stem, Leaf }\end{array}$ & $\begin{array}{l}\text { Potherb } \\
\text { cooked like } \\
\text { spinach }\end{array}$ & Not mentioned & $\begin{array}{l}\text { Not } \\
\text { mentioned }\end{array}$ & Not mentioned & Lou et al. 2019 \\
\hline $\begin{array}{l}\text { Elatostema } \\
\text { laetevirens } \\
\text { Makino }\end{array}$ & $\begin{array}{l}\text { Not } \\
\text { Collected }\end{array}$ & $\begin{array}{l}\text { CHINA, INDIA } \\
\text { (Arunachal } \\
\text { Pradesh) }\end{array}$ & $\begin{array}{l}\text { Monpa } \\
\text { community }\end{array}$ & $\begin{array}{l}\text { Not } \\
\text { mentioned }\end{array}$ & Leaves & $\begin{array}{l}\text { Tender } \\
\text { Leaves } \\
\text { cooked as } \\
\text { vegetable }\end{array}$ & Not mentioned & $\begin{array}{l}\text { Not } \\
\text { mentioned }\end{array}$ & Not mentioned & $\begin{array}{l}\text { Plants for a } \\
\text { Future } \\
\text { (https://pfaf.org/); } \\
\text { Tsering et al. } \\
2017\end{array}$ \\
\hline $\begin{array}{l}\text { Elatostema } \\
\text { laevissimum W.T. } \\
\text { Wang }\end{array}$ & $\begin{array}{l}\text { Not } \\
\text { Collected }\end{array}$ & $\begin{array}{l}\text { CHINA } \\
\text { (Yunnan) }\end{array}$ & Not mentioned & $\begin{array}{l}\text { Not } \\
\text { mentioned }\end{array}$ & $\begin{array}{l}\text { Whole } \\
\text { plant }\end{array}$ & $\begin{array}{l}\text { Not } \\
\text { mentioned }\end{array}$ & Not mentioned & $\begin{array}{l}\text { Used as } \\
\text { fodder to } \\
\text { feed } \\
\text { Mithun } \\
\text { (Bos } \\
\text { frontalis) }\end{array}$ & Not mentioned & Geng et al. 2017 \\
\hline \multirow[t]{4}{*}{$\begin{array}{l}\text { Elatostema } \\
\text { lineolatum Wight }\end{array}$} & \multirow[t]{4}{*}{71641} & \multirow{4}{*}{$\begin{array}{l}\text { INDIA } \\
\text { (Arunachal } \\
\text { Pradesh, } \\
\text { Nagaland, } \\
\text { Manipur, } \\
\text { Assam, Nilgiris) }\end{array}$} & $\begin{array}{l}\text { Monpa } \\
\text { community }\end{array}$ & Dambe-hru & \multirow[t]{4}{*}{$\begin{array}{l}\text { Shoots } \\
\text { and leaves }\end{array}$} & \multirow{4}{*}{$\begin{array}{l}\text { Tender } \\
\text { shoots and } \\
\text { leaves used } \\
\text { as } \\
\text { vegetable; } \\
\text { Boiled with } \\
\text { rice to } \\
\text { prepare } \\
\text { Galho(an } \\
\text { angami dish) }\end{array}$} & \multirow{4}{*}{$\begin{array}{l}\text { Paste of leaf } \\
\text { applied on cuts } \\
\text { caused by rocks } \\
\text { stones or iron } \\
\text { pieces }\end{array}$} & \multirow[t]{4}{*}{$\begin{array}{l}\text { Not } \\
\text { mentioned }\end{array}$} & \multirow[t]{4}{*}{ Not mentioned } & \multirow{4}{*}{$\begin{array}{l}\text { Tsering et al. } \\
\text { 2017; Singh \& } \\
\text { Teron 2015; } \\
\text { Konsam et al. } \\
\text { 2016b; Borthakur } \\
\text { 1976; Paulsamy } \\
\text { et al. } 2007\end{array}$} \\
\hline & & & Angami tribe & Jothu, Gazo & & & & & & \\
\hline & & & Manipur & Ching sougri & & & & & & \\
\hline & & & $\begin{array}{l}\text { Karbi- } \\
\text { Anglong } \\
\text { tribes }\end{array}$ & Himbu & & & & & & \\
\hline
\end{tabular}




\begin{tabular}{|c|c|c|c|c|c|c|c|c|c|c|}
\hline $\begin{array}{l}\text { Elatostema } \\
\text { longipes W.T. } \\
\text { Wang }\end{array}$ & $\begin{array}{l}\text { Not } \\
\text { Collected }\end{array}$ & THAILAND & Lua tribe & $\begin{array}{l}\text { Not } \\
\text { mentioned }\end{array}$ & Leaves & $\begin{array}{l}\text { Not } \\
\text { mentioned }\end{array}$ & $\begin{array}{l}\text { Used for treating } \\
\text { diabetes }\end{array}$ & $\begin{array}{l}\text { Not } \\
\text { mentioned }\end{array}$ & Not mentioned & $\begin{array}{l}\text { Phumthum \& } \\
\text { Balslev } 2018\end{array}$ \\
\hline \multirow{2}{*}{$\begin{array}{l}\text { Elatostema } \\
\text { macrophyllum } \\
\text { Brongn. }\end{array}$} & \multirow[t]{2}{*}{$\begin{array}{l}\text { Not } \\
\text { Collected }\end{array}$} & \multirow{2}{*}{$\begin{array}{l}\text { INDONESIA } \\
\text { (Sumbawa } \\
\text { Island), PAPUA } \\
\text { NEW GUINEA, }\end{array}$} & $\begin{array}{l}\text { Samawa } \\
\text { community }\end{array}$ & telat & \multirow[t]{2}{*}{$\begin{array}{l}\text { stems, } \\
\text { leaves }\end{array}$} & \multirow{2}{*}{$\begin{array}{l}\text { Eaten as } \\
\text { vegetable; } \\
\text { people eat } \\
\text { plant ash as } \\
\text { salt }\end{array}$} & \multirow[t]{2}{*}{ Not mentioned } & \multirow[t]{2}{*}{$\begin{array}{l}\text { Not } \\
\text { mentioned }\end{array}$} & \multirow{2}{*}{$\begin{array}{l}\text { Plant ash used } \\
\text { as salt } \\
\text { substitute }\end{array}$} & \multirow{2}{*}{$\begin{array}{l}\text { Rahayu \& } \\
\text { Rustiami 2017; } \\
\text { Rugayah et al. } \\
\text { 1989; Ohtsuka et } \\
\text { al. } 1987\end{array}$} \\
\hline & & & $\begin{array}{l}\text { Papua New } \\
\text { Guinea }\end{array}$ & Gidra & & & & & & \\
\hline $\begin{array}{l}\text { Elatostema } \\
\text { monandrum } \\
\text { (Buch.-Ham. ex } \\
\text { D.Don)H.Hara }\end{array}$ & 71645 & $\begin{array}{l}\text { NEPAL } \\
\text { (Langtang } \\
\text { Village) }\end{array}$ & Not mentioned & $\begin{array}{l}\text { Not } \\
\text { mentioned }\end{array}$ & Roots & $\begin{array}{l}\text { Not } \\
\text { mentioned }\end{array}$ & $\begin{array}{l}\text { Root paste is used } \\
\text { to treat cuts and } \\
\text { wounds }\end{array}$ & $\begin{array}{l}\text { Not } \\
\text { mentioned }\end{array}$ & Not mentioned & Shrestha 2015 \\
\hline $\begin{array}{l}\text { Elatostema } \\
\text { nasutum Hook.f. }\end{array}$ & 71605 & $\begin{array}{l}\text { CHINA and in } \\
\text { Yunnan }\end{array}$ & $\begin{array}{l}\text { Monpa } \\
\text { community }\end{array}$ & Da-mi-ru & $\begin{array}{l}\text { Stem, } \\
\text { Leaves }\end{array}$ & $\begin{array}{l}\text { Leaves used } \\
\text { as vegetable } \\
\text { which is first } \\
\text { boiled in } \\
\text { water before } \\
\text { consumption }\end{array}$ & Not mentioned & $\begin{array}{l}\text { Used as } \\
\text { fodder to } \\
\text { feed } \\
\text { Mithun } \\
\text { (Bos } \\
\text { frontalis) }\end{array}$ & $\begin{array}{l}\text { Stem used as } \\
\text { rope or twine }\end{array}$ & $\begin{array}{l}\text { Li et al. } 2020 ; \\
\text { Geng et al. } 2017\end{array}$ \\
\hline $\begin{array}{l}\text { Elatostema } \\
\text { paivaeanum } \\
\text { Wedd. }\end{array}$ & $\begin{array}{l}\text { Not } \\
\text { Collected }\end{array}$ & $\begin{array}{l}\text { TANZANIA } \\
\text { (Kilimanjaro) }\end{array}$ & Chagga & $\begin{array}{l}\text { nzunga, } \\
\text { isunguwala, } \\
\text { lya muringrni }\end{array}$ & $\begin{array}{l}\text { Not } \\
\text { mentioned }\end{array}$ & $\begin{array}{l}\text { Not } \\
\text { mentioned }\end{array}$ & $\begin{array}{l}\text { gastro-intestinal } \\
\text { remedies }\end{array}$ & $\begin{array}{l}\text { Not } \\
\text { mentioned }\end{array}$ & Not mentioned & Hemp 1999 \\
\hline $\begin{array}{l}\text { Elatostema } \\
\text { papillosum Wedd. }\end{array}$ & 71617 & $\begin{array}{l}\text { BANGLADESH } \\
\text { (Hill tracts) }\end{array}$ & Chakma tribe & $\begin{array}{l}\text { Not } \\
\text { mentioned }\end{array}$ & $\begin{array}{l}\text { Stems and } \\
\text { Leaves }\end{array}$ & $\begin{array}{l}\text { Not } \\
\text { mentioned }\end{array}$ & $\begin{array}{l}\text { Extract of stems } \\
\text { and leaves is } \\
\text { given to drink for } \\
\text { treating hysteria, } \\
\text { abdominal pain }\end{array}$ & $\begin{array}{l}\text { Not } \\
\text { mentioned }\end{array}$ & Not mentioned & $\begin{array}{l}\text { Rahman et al. } \\
2007\end{array}$ \\
\hline $\begin{array}{l}\text { Elatostema } \\
\text { parasiticum Blume } \\
\text { ex H. Schroet. }\end{array}$ & $\begin{array}{l}\text { Not } \\
\text { Collected }\end{array}$ & INDONESIA & $\begin{array}{l}\text { Siwai and } \\
\text { Buin tribe }\end{array}$ & $\begin{array}{l}\text { Not } \\
\text { mentioned }\end{array}$ & $\begin{array}{l}\text { Not } \\
\text { mentioned }\end{array}$ & $\begin{array}{l}\text { Not } \\
\text { mentioned }\end{array}$ & $\begin{array}{l}\text { Used for treating } \\
\text { fever, shows anti- } \\
\text { microbial activity }\end{array}$ & $\begin{array}{l}\text { Not } \\
\text { mentioned }\end{array}$ & Not mentioned & $\begin{array}{l}\text { Mariani et al. } \\
\text { 2016; Stefan \& } \\
\text { Birsa } 2019\end{array}$ \\
\hline $\begin{array}{l}\text { Elatostema } \\
\text { parvum } \\
\text { (Blume)Blume ex } \\
\text { Miq. }\end{array}$ & 73752 & INDIA (Assam) & $\begin{array}{l}\text { Karbi and } \\
\text { Munda } \\
\text { communities }\end{array}$ & $\begin{array}{l}\text { Longle } \\
\text { mehek }\end{array}$ & Leaves & $\begin{array}{l}\text { Young } \\
\text { leaves are } \\
\text { eaten with } \\
\text { pulses }\end{array}$ & Not mentioned & $\begin{array}{l}\text { Not } \\
\text { mentioned }\end{array}$ & Not mentioned & Borah et al. 2020 \\
\hline \multirow{4}{*}{$\begin{array}{l}\text { Elatostema } \\
\text { platyphyllum } \\
\text { Wedd. }\end{array}$} & \multirow[t]{4}{*}{71640} & \multirow{4}{*}{$\begin{array}{l}\text { INDIA } \\
\text { (Arunachal } \\
\text { Pradesh, } \\
\text { Assam, Sikkim); }\end{array}$} & Nyshi tribe & Huj & \multirow{4}{*}{$\begin{array}{l}\text { Roots, } \\
\text { Leaves, } \\
\text { Whole } \\
\text { plant }\end{array}$} & \multirow{4}{*}{$\begin{array}{l}\text { Leaves used } \\
\text { as } \\
\text { vegetable, }\end{array}$} & \multirow{4}{*}{$\begin{array}{l}\text { Roots juice used } \\
\text { for inducing } \\
\text { vomiting; Paste of } \\
\text { leaves applied on }\end{array}$} & \multirow{4}{*}{$\begin{array}{l}\text { Whole } \\
\text { plant is } \\
\text { used as } \\
\text { forage to }\end{array}$} & \multirow[t]{4}{*}{ Not mentioned } & \multirow{4}{*}{$\begin{array}{l}\text { Murtem \& } \\
\text { Chaudhry 2016a; } \\
\text { Murtem \& } \\
\text { Chaudhry 2016b; }\end{array}$} \\
\hline & & & Galo tribe & Hoj Ao & & & & & & \\
\hline & & & Adi tribe & Sakobadha & & & & & & \\
\hline & & & Apatani tribe & Hiipe & & & & & & \\
\hline
\end{tabular}




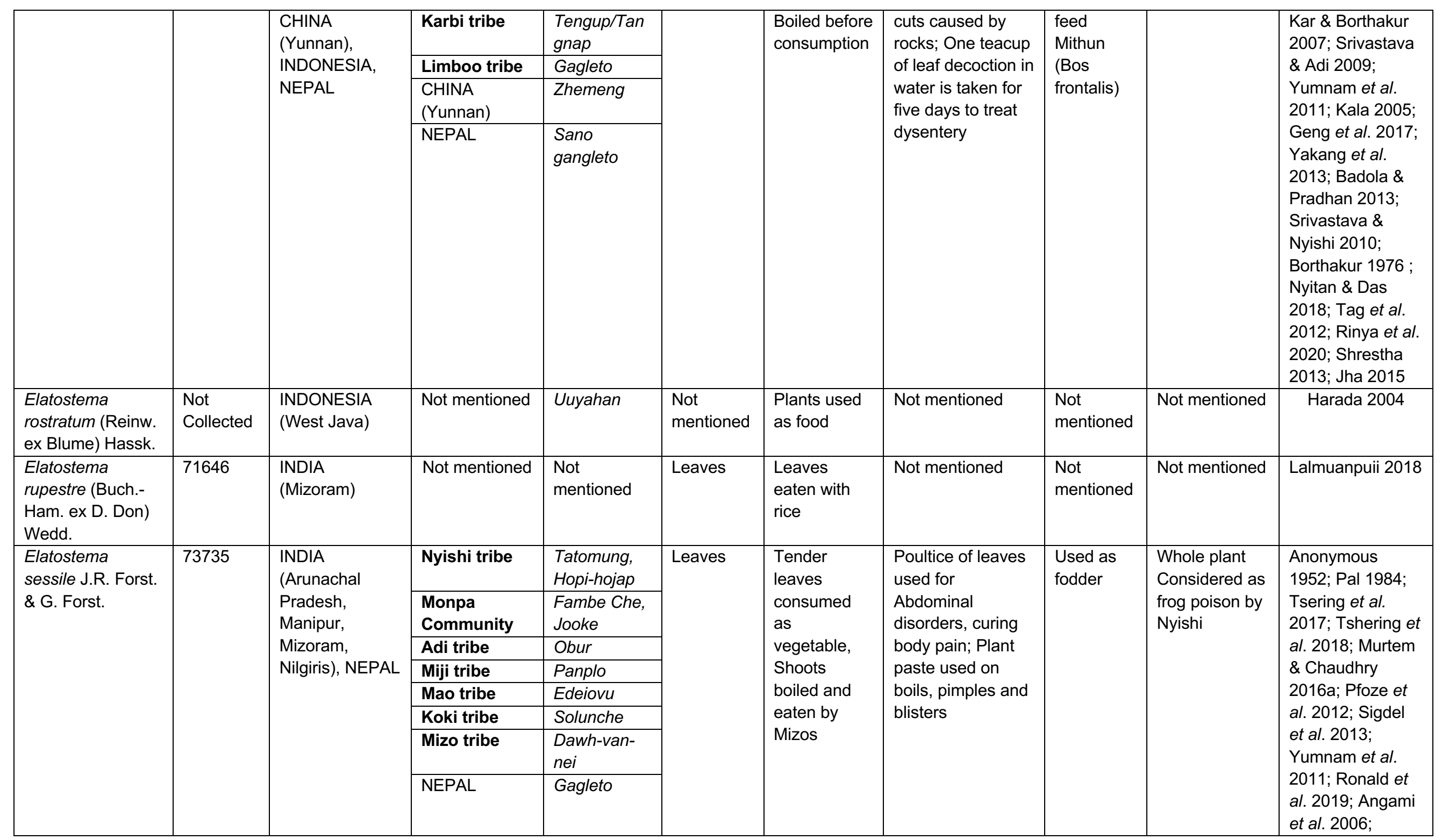




\begin{tabular}{|c|c|c|c|c|c|c|c|c|c|c|}
\hline & & & & & & & & & & $\begin{array}{l}\text { Muthu \& Rimo } \\
\text { 2018; Srivastava } \\
\text { \& Nyishi 2010; } \\
\text { Kar et al. 2013; } \\
\text { Paulsamy et al. } \\
\text { 2007; Gewali } \\
\text { 2008; Shrestha } \\
\text { 2013; Saha \& } \\
\text { Sundriyal 2013 }\end{array}$ \\
\hline $\begin{array}{l}\text { Elatostema } \\
\text { strigosum Hassk. }\end{array}$ & $\begin{array}{l}\text { Not } \\
\text { Collected }\end{array}$ & $\begin{array}{l}\text { INDONESIA } \\
\text { (North Sumatra) }\end{array}$ & $\begin{array}{l}\text { Sub-ethnic } \\
\text { Batak } \\
\text { Simalungun }\end{array}$ & Sisik naga & $\begin{array}{l}\text { Leaves, } \\
\text { fruits }\end{array}$ & $\begin{array}{l}\text { Not } \\
\text { mentioned }\end{array}$ & $\begin{array}{l}\text { Used for treating } \\
\text { hypertension, } \\
\text { Injury, } \\
\text { Gastrointestinal } \\
\text { disorders and } \\
\text { fever }\end{array}$ & $\begin{array}{l}\text { Not } \\
\text { mentioned }\end{array}$ & Not mentioned & $\begin{array}{l}\text { Silalahi et al. } \\
\text { 2015; Purba et al. } \\
2016\end{array}$ \\
\hline $\begin{array}{l}\text { Elatostema } \\
\text { umbellatum } \\
\text { (Siebold \& Zucc.) } \\
\text { Blume }\end{array}$ & $\begin{array}{l}\text { Not } \\
\text { Collected }\end{array}$ & $\begin{array}{l}\text { JAPAN } \\
\text { (Irouzaki) }\end{array}$ & & Uwabamiso & $\begin{array}{l}\text { Not } \\
\text { mentioned }\end{array}$ & $\begin{array}{l}\text { Food. Used } \\
\text { as side dish }\end{array}$ & Not mentioned & $\begin{array}{l}\text { Not } \\
\text { mentioned }\end{array}$ & Not mentioned & Wiley et al. 2019 \\
\hline Elatostema sp 1. & $\begin{array}{l}\text { Not } \\
\text { Collected }\end{array}$ & $\begin{array}{l}\text { INDONESIA } \\
\text { (Central } \\
\text { Sulawesi) }\end{array}$ & $\begin{array}{l}\text { Kaili Inde } \\
\text { tribe }\end{array}$ & $\begin{array}{l}\text { Pedura } \\
\text { walehu }\end{array}$ & $\begin{array}{l}\text { Not } \\
\text { mentioned }\end{array}$ & $\begin{array}{l}\text { Not } \\
\text { mentioned }\end{array}$ & Skin disease & $\begin{array}{l}\text { Not } \\
\text { mentioned }\end{array}$ & Not mentioned & $\begin{array}{l}\text { Fathurrahman et } \\
\text { al. } 2016\end{array}$ \\
\hline Elatostema sp 2. & $\begin{array}{l}\text { Not } \\
\text { Collected }\end{array}$ & $\begin{array}{l}\text { PAPUA NEW } \\
\text { GUINEA (East } \\
\text { Sepik Province) }\end{array}$ & Not mentioned & $\begin{array}{l}\text { Kaskas- } \\
\text { Bhirs }\end{array}$ & $\begin{array}{l}\text { Not } \\
\text { mentioned }\end{array}$ & $\begin{array}{l}\text { Not } \\
\text { mentioned }\end{array}$ & Scabies & $\begin{array}{l}\text { Not } \\
\text { mentioned }\end{array}$ & Not mentioned & Koch et al. 2015 \\
\hline Elatostema sp 3. & $\begin{array}{l}\text { Not } \\
\text { Collected }\end{array}$ & $\begin{array}{l}\text { PAPUA NEW } \\
\text { GUINEA (East } \\
\text { Sepik Province) }\end{array}$ & Not mentioned & Moin Kukuri & $\begin{array}{l}\text { Not } \\
\text { mentioned }\end{array}$ & $\begin{array}{l}\text { Not } \\
\text { mentioned }\end{array}$ & $\begin{array}{l}\text { Fever, Headache, } \\
\text { Joint Pain, Fertility }\end{array}$ & $\begin{array}{l}\text { Not } \\
\text { mentioned }\end{array}$ & Not mentioned & Koch et al. 2015 \\
\hline Elatostema sp 4. & $\begin{array}{l}\text { Not } \\
\text { Collected }\end{array}$ & $\begin{array}{l}\text { SUMATRA } \\
\text { (Samosir } \\
\text { district) }\end{array}$ & $\begin{array}{l}\text { Batak Toba } \\
\text { community }\end{array}$ & Aup-Aup & $\begin{array}{l}\text { Not } \\
\text { mentioned }\end{array}$ & $\begin{array}{l}\text { Not } \\
\text { mentioned }\end{array}$ & Medicinal & $\begin{array}{l}\text { Not } \\
\text { mentioned }\end{array}$ & Not mentioned & $\begin{array}{l}\text { Ibo \& Arimukti } \\
2019\end{array}$ \\
\hline Elatostema sp 5. & $\begin{array}{l}\text { Not } \\
\text { Collected }\end{array}$ & $\begin{array}{l}\text { INDIA (Manipur- } \\
\text { Ukhrul district) }\end{array}$ & $\begin{array}{l}\text { Tangkhul } \\
\text { tribe }\end{array}$ & Hantekhan & Leaves & $\begin{array}{l}\text { Cooked with } \\
\text { meat or dry } \\
\text { fish }\end{array}$ & Not mentioned & $\begin{array}{l}\text { Not } \\
\text { mentioned }\end{array}$ & Not mentioned & Salam et al. 2012 \\
\hline Elatostema sp 6. & $\begin{array}{l}\text { Not } \\
\text { Collected }\end{array}$ & $\begin{array}{l}\text { Papua New } \\
\text { Guinea }\end{array}$ & $\begin{array}{l}\text { Ndumba } \\
\text { Tribe }\end{array}$ & Merura & Leaves & $\begin{array}{l}\text { Not } \\
\text { mentioned }\end{array}$ & $\begin{array}{l}\text { Leaves eaten as } \\
\text { medicine }\end{array}$ & & $\begin{array}{l}\text { Leaves used } \\
\text { as }\end{array}$ & Hays 1980 \\
\hline
\end{tabular}




\begin{tabular}{|c|c|c|c|c|c|c|c|c|c|c|}
\hline & & & & & & & & & $\begin{array}{l}\text { ornamentation } \\
\text { by women }\end{array}$ & \\
\hline Elatostema sp 7. & $\begin{array}{l}\text { Not } \\
\text { Collected }\end{array}$ & $\begin{array}{l}\text { Papua New } \\
\text { Guinea }\end{array}$ & $\begin{array}{l}\text { Ndumba } \\
\text { Tribe }\end{array}$ & $\begin{array}{l}\text { Nronggaaria } \\
\text { ara }\end{array}$ & Leaves & $\begin{array}{l}\text { Not } \\
\text { mentioned }\end{array}$ & $\begin{array}{l}\text { Leaves eaten as } \\
\text { medicine }\end{array}$ & $\begin{array}{l}\text { Leaves } \\
\text { fed to pigs } \\
\text { to } \\
\text { incraese } \\
\text { their body } \\
\text { fat }\end{array}$ & $\begin{array}{l}\text { Leaves eaten } \\
\text { on ceremonies }\end{array}$ & Hays 1980 \\
\hline Elatostema sp 8. & $\begin{array}{l}\text { Not } \\
\text { Collected }\end{array}$ & $\begin{array}{l}\text { INDONESIA } \\
\text { (Sumatra) }\end{array}$ & Not mentioned & Kerih & Leaves & $\begin{array}{l}\text { Not } \\
\text { mentioned }\end{array}$ & $\begin{array}{l}\text { Leaves used in } \\
\text { fever }\end{array}$ & $\begin{array}{l}\text { Not } \\
\text { mentioned }\end{array}$ & Not mentioned & $\begin{array}{l}\text { Silalahi et al. } \\
2018\end{array}$ \\
\hline Elatostema sp 9. & $\begin{array}{l}\text { Not } \\
\text { Collected }\end{array}$ & $\begin{array}{l}\text { INDONESIA } \\
\text { (Sumatra) }\end{array}$ & Not mentioned & Komil & Leaves & $\begin{array}{l}\text { Not } \\
\text { mentioned }\end{array}$ & Not mentioned & $\begin{array}{l}\text { Not } \\
\text { mentioned }\end{array}$ & Not mentioned & $\begin{array}{l}\text { Silalahi et al. } \\
2018\end{array}$ \\
\hline Elatostema sp 10. & $\begin{array}{l}\text { Not } \\
\text { Collected }\end{array}$ & $\begin{array}{l}\text { Papua New } \\
\text { Guinea (Kalam, } \\
\text { Upper Kaironk } \\
\text { valley) }\end{array}$ & Kalam People & Gangal & $\begin{array}{l}\text { Not } \\
\text { mentioned }\end{array}$ & $\begin{array}{l}\text { Not } \\
\text { mentioned }\end{array}$ & Not mentioned & $\begin{array}{l}\text { Not } \\
\text { mentioned }\end{array}$ & $\begin{array}{l}\text { Used for } \\
\text { beauty magic }\end{array}$ & Gardner 2010 \\
\hline Elatostema sp 11. & $\begin{array}{l}\text { Not } \\
\text { Collected }\end{array}$ & $\begin{array}{l}\text { Papua New } \\
\text { Guinea (Kalam, } \\
\text { Upper Kaironk } \\
\text { valley) }\end{array}$ & Kalam People & Sspi-lum-ket & $\begin{array}{l}\text { Not } \\
\text { mentioned }\end{array}$ & $\begin{array}{l}\text { Not } \\
\text { mentioned }\end{array}$ & Not mentioned & $\begin{array}{l}\text { Not } \\
\text { mentioned }\end{array}$ & $\begin{array}{l}\text { worn in belt } \\
\text { and arm belts } \\
\text { as decoration }\end{array}$ & Gardner 2010 \\
\hline Elatostema sp 12. & $\begin{array}{l}\text { Not } \\
\text { Collected }\end{array}$ & $\begin{array}{l}\text { Papua New } \\
\text { Guinea (Kalam, } \\
\text { Upper Kaironk } \\
\text { valley) }\end{array}$ & Kalam People & Yuley & Shoots & $\begin{array}{l}\text { Shoots eaten } \\
\text { raw }\end{array}$ & Not mentioned & $\begin{array}{l}\text { Not } \\
\text { mentioned }\end{array}$ & $\begin{array}{l}\text { Foliage used } \\
\text { in beauty } \\
\text { magic }\end{array}$ & Gardner 2010 \\
\hline Elatostema sp 13. & $\begin{array}{l}\text { Not } \\
\text { Collected }\end{array}$ & $\begin{array}{l}\text { Solomon } \\
\text { Islands }\end{array}$ & Not mentioned & $\begin{array}{l}\text { Not } \\
\text { mentioned }\end{array}$ & $\begin{array}{l}\text { Not } \\
\text { mentioned }\end{array}$ & $\begin{array}{l}\text { Not } \\
\text { mentioned }\end{array}$ & $\begin{array}{l}\text { Plants thought to } \\
\text { give life, health } \\
\text { and strength by } \\
\text { Islanders }\end{array}$ & $\begin{array}{l}\text { Not } \\
\text { mentioned }\end{array}$ & Not mentioned & $\begin{array}{l}\text { Tedder \& Tedder } \\
1979\end{array}$ \\
\hline
\end{tabular}




\section{Data analysis}

Ethnobotanical data were categorized as plant parts used, usage as food, medicinal uses, forage and others (ornamental, material uses etc.). The food category comprises of species that are used for human consumption; the medicinal category comprises of species used in varied forms for healing and therapeutical effects; the forage category comprises of species collected from the wild especially for feeding domesticated animals; finally, the others category comprises of species used for ornamentation, counter-magic and material purposes such as making ropes.

To assess if there is any significant difference in leaf area regarding type of use (Human consumption, Medicinal use, Forage, Other use) we used HO (null hypothesis) and $\mathrm{H} 1$ (our hypothesis)

$\mathrm{HO}$ : There is no significant difference in leaf area regarding type of use.

$\mathrm{H} 1$ : There is a significant difference in leaf area regarding type of use.

Our data set had two independent categorical variables (1. Use, 2. Status) and one dependent numerical variable (Leaf area). Variable use has four levels (Human-consumption, Medicinal-use, Forage, Other-use) and variable status has two levels (Recorded, Not-recorded). Groups in variable use (Human-consumption, Medicinal-use, Forage, Other-use) are treated as main groups and groups in variable status are treated as subgroups of the main. First the data was visually analyzed. Shapiro-Wilks test was performed to test normality. As all the data were found to be non-parametric, Kruskal-Wallis rank sum test was done for analyzing significant difference. For better understanding of how all parameters correlate and the data distribution Principal Component Analysis (PCA) was done. Statistical analysis and making of plots were done using $\mathrm{R}$ studio3.0.

\section{Use-value (UV)}

Ethnobotanical studies seek to identify important plant species for a given culture. In order to make conclusions about plant-human knowledge across cultures we must be able to measure ethnobotanical knowledge in a uniform way (Zenderland et al. 2019). Use-value index is widely used for the purpose of quantifying the importance of useful plants (Phillips \& Gentry 1993). Formula given by Rossato et al. (1999) $\left(U V=\sum U_{i} / n\right)$ is used for these studies. $U_{i}$ is total number of uses recorded for a species while, $n$ is total number of informants or frequency of citation. We used Use-Value Index across recorded categories (Food, Medicinal, Forage and Others) to find out the relation between number of recorded uses per species and their actual Use value in our datasets.

\section{Results}

Ethnobotanical analysis of Elatostema species

We identified 30 indigenous communities from all over the globe that use Elatostema species. From India 15 indigenous communities were recorded and 15 were recorded from other countries. One indigenous community, Monpa was seen to exist both in India and China (Table 1).

Of the ca. 500 Elatostema species in the world, we identified 40 taxa that have documented ethnobotanical usage (Table 2). For human consumption 16 species were used, 20 species were used for medicinal purposes, seven species were used as forage and ten species had varied (ornamental/material) usage. We observed that most of the documented species had their native ranges in South Asia, East Asia and South East Asia (Table 3) which corresponds with the fact that the center for diversity of Elatostema species is towards these regions (Hadiah et al. 2003; Hadiah \& Conn 2009; Wei et al. 2011; Wu et al. 2013; Tseng et al. 2019).

Five indigenous communities use more than one Elatostema species. Monpa-Arunachal Pradesh (4 species), Kalam- Papua New Guinea (3 species), Nyishi- Arunachal Pradesh (2 species), AdiArunachal Pradesh (2 species) and Ndumba- Papua New Guinea (2 species). Moreover, four species (Elatostema sessile J.R. Forster \& G. Forster, Elatostema lineolatum Wight, Elatostema platyphyllum Wedd. and Elatostema macrophyllum Brongn.) in our studies were used by more than one indigenous group.

\section{Elatostema species used as medicine}

The most common Ethnobotanical usage of Elatostema species in our study is as medicine. In our study 20 Elatostema species were used as medicine (Table 2). Extracts from stems and leaves, leaf decoction and poultice of leaves of five species viz. E. sessile, E. platyphyllum, E. papillosum, E. paivaeanum and $E$. strigosum were used to treat gastrointestinal disorders and abdominal pain by indigenous communities in India, China, Indonesia, Nepal, Tanzania and Bangladesh (Table 2). Six species viz. E. integrifolium E. strigosum, $E$. parasiticum, E. ficoides, E. sp. 3 and E. sp. 6 were used to treat fever. Paste of leaves and stem of three Elatostema species viz. $E$. lineolatum, $E$. platyphyllum and $E$. monandrum were used to treat cuts and wounds caused by rocks and iron pieces.

Plant paste of $E$. sessile was used to treat boils, pimples and blisters by Nyishis, Monpas and Adis of 
Arunachal Pradesh, India. Extracts of stems and leaves of $E$. papillosum were used to treat hysteria by Chakma in Bangladesh. Leaves of $E$. strigosum were used in treating hypertension by Batak Simalungun in Indonesia. E. longipes was recorded to be used in treating diabetes by Lua community in Thailand (Phumthum \& Balslev 2018). Leaf juice of $E$. heyneanum were used for treating immunodeficiency in children. E. sp. 13 of Solomon
Islands was recorded to give life, health and strength to Islanders (Tedder \& Tedder 1979).

In many cases such as E. paivaeanum, E. strigosum, E. longipes, E. parasiticum, E. sp. 1, E. sp. 2, E. sp. 3, E. sp. 4, E. sp. 6, E. sp. 7, E. sp. 8, and E. sp. 13 recorded for medicinal usage in our study there were no description of preparation methods. We recorded 15 species in our study that had exclusive medicinal usage (no food, forage and other uses recorded).

Table 3. Native ranges of recorded Elatostema species. Data collected from POWO (Plants of the world online) and recorded localities in published reports.

\begin{tabular}{|c|c|}
\hline Species & Native range \\
\hline Elatostema dissectum Wedd. & South Asia, East Asia \\
\hline Elatostema cuneatum Wight & South Asia, East Asia, South East Asia \\
\hline Elatostema laetevirens Makino & East Asia \\
\hline Elatostema sessile J.R. Forst. \& G. Forst. & South Asia, East Asia, South East Asia \\
\hline Elatostema integrifolium (D. Don) Wedd. & South Asia, East Asia, South East Asia \\
\hline Elatostema lineolatum Wight & South Asia, East Asia, South East Asia \\
\hline Elatostema beccarii H.Schroet. & Papua New Guinea, Solomon Islands \\
\hline Elatostema platyphyllum Wedd. & South Asia, East Asia, South East Asia \\
\hline Elatostema cuneiforme W.T. Wang & East Asia \\
\hline Elatostema nasutum Hook.f. & South Asia, East Asia, South East Asia \\
\hline Elatostema paivaeanum Wedd. & Africa \\
\hline Elatostema umbellatum (Siebold \& Zucc.) Blume & East Asia \\
\hline Elatostema papillosum Wedd. & South Asia, South East Asia \\
\hline Elatostema strigosum Hassk. & South East Asia \\
\hline Elatostema acuminatum (Poir.) Brongn. & South Asia, East Asia, South East Asia \\
\hline Elatostema longipes W.T. Wang & East Asia \\
\hline Elatostema involucratum Franch. \& Sav. & East Asia \\
\hline Elatostema parasiticum Blume ex H. Schroet. & South East Asia \\
\hline Elatostema ficoides Wedd. & South Asia, East Asia, South East Asia \\
\hline Elatostema parvum (Blume) Blume ex Miq. & South Asia, East Asia, South East Asia \\
\hline Elatostema hookerianum Wedd. & South Asia, East Asia, South East Asia \\
\hline Elatostema laevissimum W.T. Wang & East Asia, South East Asia \\
\hline Elatostema macrophyllum Brongn. & South East Asia \\
\hline Elatostema rupestre (Buch.-Ham. ex D. Don)Wedd. & South Asia, East Asia, South East Asia \\
\hline Elatostema monandrum (Buch. -Ham. ex D. Don) H. Hara & South Asia, East Asia, South East Asia \\
\hline Elatostema rostratum (Reinw. ex Blume) Hassk. & South Asia, South East Asia \\
\hline Elatostema heyneanum (Wedd.) Hallier $\mathrm{f}$. & South Asia, East Asia, South East Asia \\
\hline Elatostema sp 1. & South East Asia \\
\hline Elatostema sp 2. & South East Asia \\
\hline Elatostema sp 3. & South East Asia \\
\hline Elatostema sp 4. & South East Asia \\
\hline Elatostema sp 5. & South Asia \\
\hline Elatostema sp 6. & South East Asia \\
\hline Elatostema sp 7. & South East Asia \\
\hline Elatostema sp 8. & South East Asia \\
\hline Elatostema sp 9. & South East Asia \\
\hline Elatostema sp 10. & South East Asia \\
\hline Elatostema sp 11. & South East Asia \\
\hline Elatostema sp 12. & South East Asia \\
\hline Elatostema sp 13. & South East Asia \\
\hline
\end{tabular}




\section{Elatostema species used for food}

In the current study we record a total of 16 Elatostema species used by various indigenous communities as food and vegetable (Table 2). In most of the cases leaves of $E$. dissectum, E. sessile, E. lineolatum, E. platyphyllum, E. nasutum, were boiled before consumption. Leaves of $E$. laetevirens, E. lineolatum, E. acuminatum, E. macrophyllum and E. sp. 5 were cooked and used as a vegetable.

Shoots and leaves of $E$. lineolatum were boiled with rice to prepare "Galho", an Angami dish in Nagaland, India. E. umbellatum was recorded to be used as a side dish in our study. E. involucratum was cooked like a pot herb by Hani community in China. It was recorded that plant ash of $E$. macrophyllum was used as a salt substitute by Samawa community in Indonesia. Leaves of E. sp. 5 was cooked with meat or dry fish by the Tangkhuls of Manipur, India. 10 species in our studies were exclusively used as food (no medicinal, forage and other uses recorded).

\section{Elatostema species as forage}

Either the leaves or whole plants of seven species viz. E. sessile, E. platyphyllum, E. cuneiforme, E. nasutum, E. hookerianum, E. laevissimum and E. sp. 7 were recorded to be used by 11 Indian indigenous communities (Nyishi, Monpa, Adi, Miji, Mao, Koki, Mizo, Galo, Apatani, Karbi, Limboo) and Ndumba tribe of Papua New Guinea in our study. E. sessile, E. platyphyllum, E. nasutum, E. hookerianum and $E$. laevissimum were used as fodder to feed Mithun (Bos frontalis) in India, Nepal and China while leaves of E. sp.7 was used to feed pigs to increase their body fat by Ndumba community in Papua New Guinea (Table 2). In our study two species namely, E. hookerianum and E. laevissimum were recorded to be exclusively used as fodder by indigenous communities in China.

\section{Elatostema species used for other purposes}

In our study we recorded 10 Elatostema species used by six indigenous communities (Nyishi, Melpa, Monpa, Samawa, Ndumba and Kalam) for ornamental, material and magical purposes (Table 2).

E. sessile was found to be used as a frog poison by Nyishis (Arunachal Pradesh, India) (Srivastava \& Nyishi, 2010); E. beccarii was recorded to be used for "counter magic" purposes by Melpas (Papua New Guinea) (Telban 1988). It is believed that if a person is possessed by a bush spirit demon or by a ghost, a healer uses leaves of $E$. beccarii, Angiopteris sp. and Piper wichmannii C. DC. which he ties up using a vine and then cooks them and rubs them over the skin of the possessed person to ward off the evil spirit (Telban 1988). Earth ovens are covered in the bottom by $E$. beccarii leaves and it is believed that this practice makes sure that their crops grow well, the men remain healthy and have a long life (Telban 1988).

$E$. cuneiforme and $E$. nasutum were used as rope or twine by Monpas (China). Plant ash of $E$. macrophyllum was used as a salt substitute by individuals of Samawa community (Sumbawa Island) E. sp. 6 and E. sp. 11 were used for ornamental and decoration purposes by Ndumbas and Kalams (Papua New Guinea).

\section{Leaf area measurements}

The usage of Wild leafy vegetables is decreasing around the world which has happened due to loss of knowledge systems needed for collection and preparation using wild plants and government development policies (Powell et al. 2014). In normal times no household depends entirely on wild edible plants for their nutrition throughout the year, but their collection and usage increase in unconventional times of food shortage and famine (Samant \& Dhar 1997; Lescure et al. 1997). Leafy wild edible plants are crucial with a view of decreasing the lacuna in traditional knowledge and extracting the potential for their utilization (Konsam et al. 2016a). We were interested in knowing if the leaf area of the Elatostema species had any relation with its usage.

From our primary visual statistical leaf area analysis, we found that, leaf area for human consumption in both recorded and not recorded groups have highest data density within 0 to $50 \mathrm{~cm}^{2}$, not recorded group has higher data density than recorded group (Figure2 ). Leaf area for medicinal use has similar kind of data density in both recorded and not recorded group, though not recorded group has highest data density between 1 to $50 \mathrm{~cm}^{2}$ and recorded group has data density around $50 \mathrm{~cm}^{2}$ (Figure-2). Data in recorded and not recorded group in case of forage seems quite differently distributed, highest data density in not recorded group is between 0 to $50 \mathrm{~cm}^{2}$, and in case of recorded group is around $50 \mathrm{~cm}^{2}$. In case of other use, the leaf area in recorded and not recorded group data density and distribution seems to vary quite highly, data density of not recorded group is highest between 0 to $50 \mathrm{~cm}^{2}$, and in recorded group 10 to $90 \mathrm{~cm}^{2}$ (Figure 2).

Data in Recorded and Not-recorded group for all the leaf use type ranges from 4.04 to $182.75 \mathrm{~cm}^{2}$, average leaf area in Recorded group for all the usage type ranges from 52.18 to $86.90 \mathrm{~cm}^{2}$ and in Not-recorded group ranges from 38.60 to $43.35 \mathrm{~cm}^{2}$, high data density in Recorded group ranges within 0 to $100 \mathrm{~cm}^{2}$, and in Not-recorded group ranges within 0 to $50 \mathrm{~cm}^{2}$ (Figure 3 ). 
From Shapiro-Wilks test data in group Human consumption ( $p$-value $=0.0005286)$, Medicinal use $(\mathrm{p}$-value $=0.0005286)$, Forage $(\mathrm{p}$-value $=$ $0.0005286)$ and Other use ( $p$-value $=0.0005286)$ were not found to be normally distributed (alpha = 0.05).

According to Kruskal-Wallis test (test for analysis of variance) there is no significant difference in data, between the groups. Human consumption, Medicinal use, Forage, Other use ( $d f=3, p$-value $=1$ ), and between the subgroups (Recorded and Not recorded) within groups viz. Human consumption (df $=1, p$-value $=0.7158)$, medicinal purpose $(\mathrm{df}=1, \mathrm{p}$ value $=0.09219)$, foraging $(\mathrm{df}=1, p$-value $=0.2433)$, other purpose $(d f=1, p$-value $=0.3653)($ Figure 4$)$.

When groups of leaf use (Human-consumption, Medicinal-use, Forage, Other-use) used as factors for performing PCA, component 1 and 2 explains about $59 \%$ of variation in data and when subgroups within the main groups (Recorded, Not recorded)

A

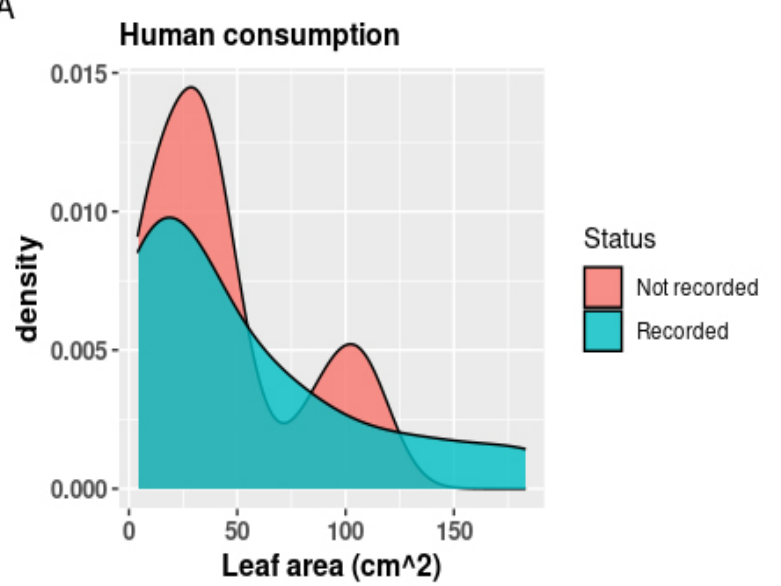

C

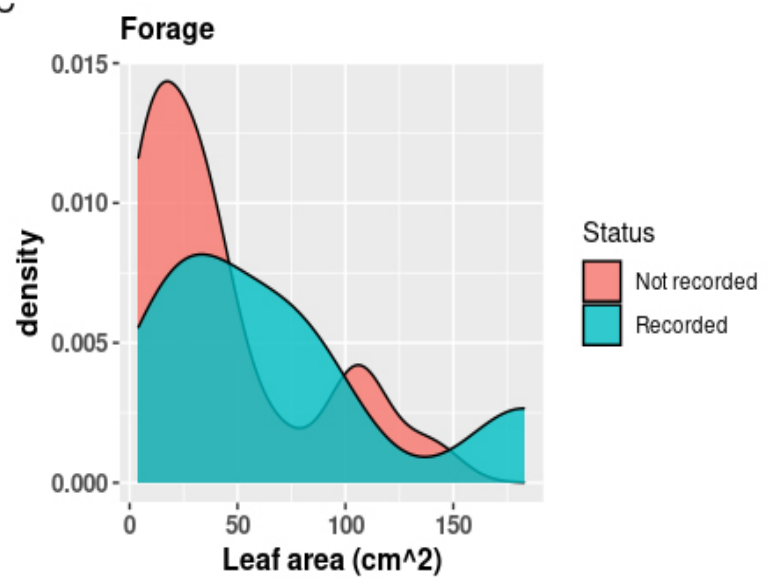

used as factors for performing PCA, component 1 and 2 explains $100 \%$ of variation in data. There is a little data clustering observed but that is quite discrete and there is high overlapping of data clusters (Figure 5).

After performing all the analysis (density plots, Shapiro-Wilks test, Kruskal-Wallis test and Principal Component Analysis) we found out that there is no significant variation in leaf area with the type of use. We did not perform a regression analysis because for a meaningful regression analysis we need two numeric variables of equal variance in all groups (in our data set there is only one numeric variable, i.e., leaf area and number of observations for all groups in our data set is highly unequal). Detailed analysis of characters such as inflorescence size, plant height and abundance may provide some insights regarding species selection for type of use in Elatostema and related leafy wild plants of interest.

B

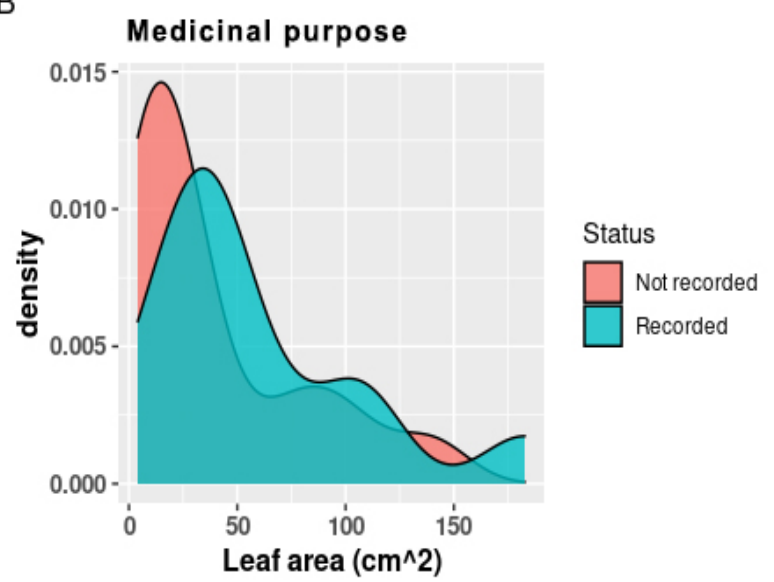

D

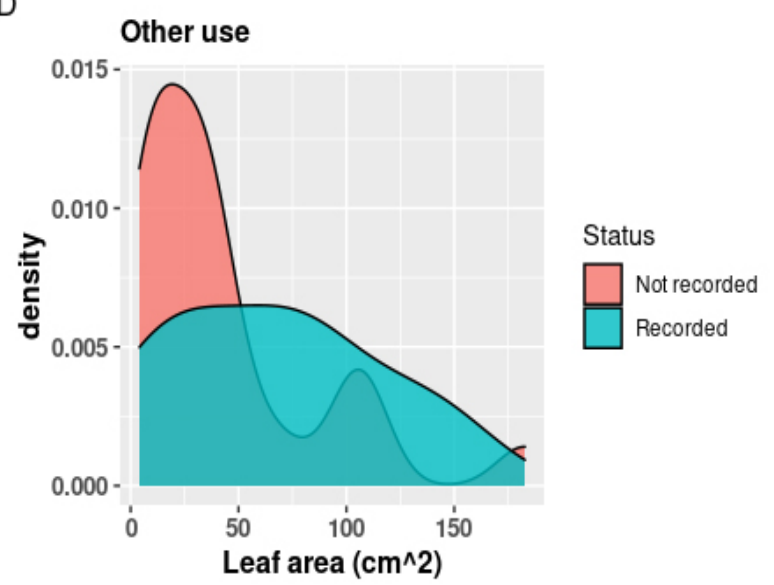

Figure 2. Density plot of leaf area regarding leaf use describing the data density in each group. 


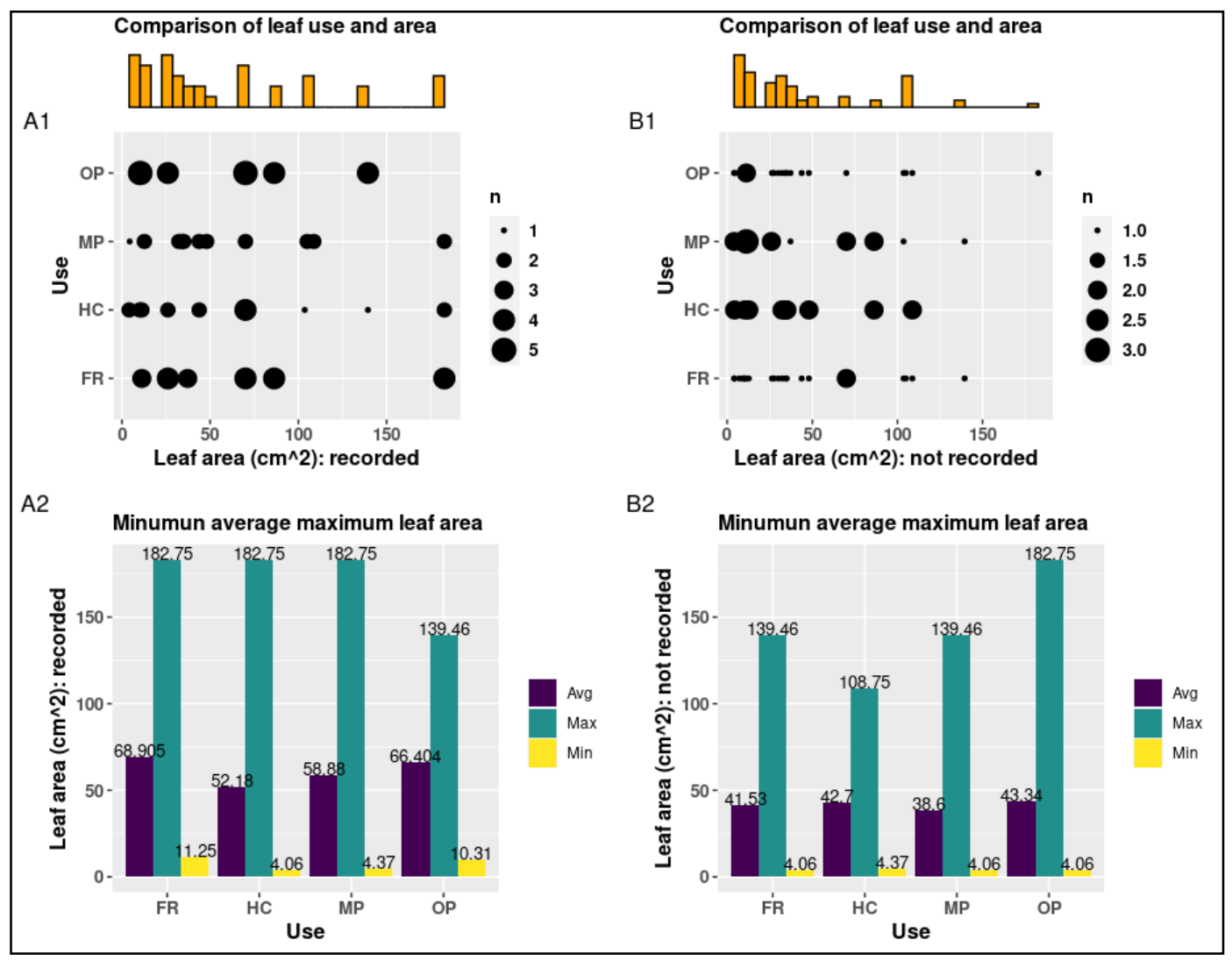

Figure 3. Visual analysis of parameters describing a comparative data density main and subgroups (A1, B1) and maximum, minimum, average leaf area in each main and subgroups (A2, B2) $[F R=$ Forage, HC = Human consumption, MP = Medicinal use, $\mathrm{OP}=$ Other use].

\section{Comparison of variation within and between groups}

A

Status 官 Not recorded 官 Recorded

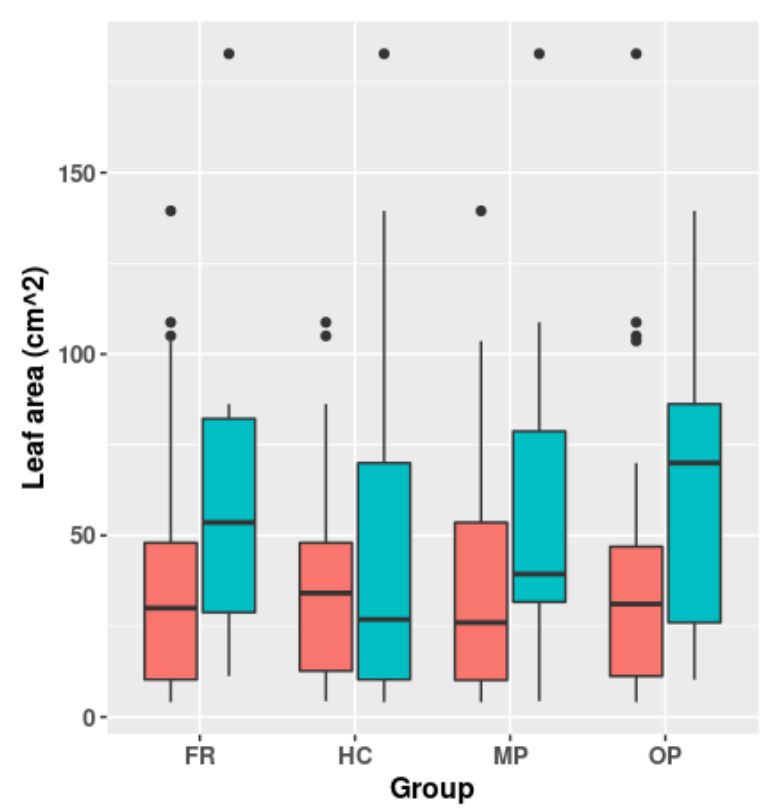

B

Group 追 FR 追 HC 审 MP 追 OP

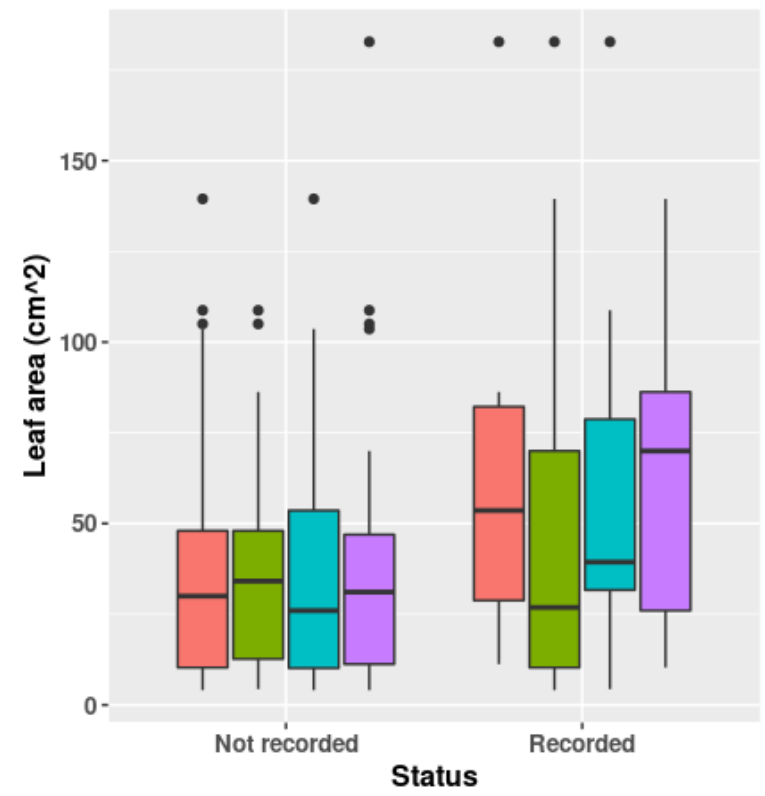

Figure 4. Boxplot showing variation in leaf area within and between groups 


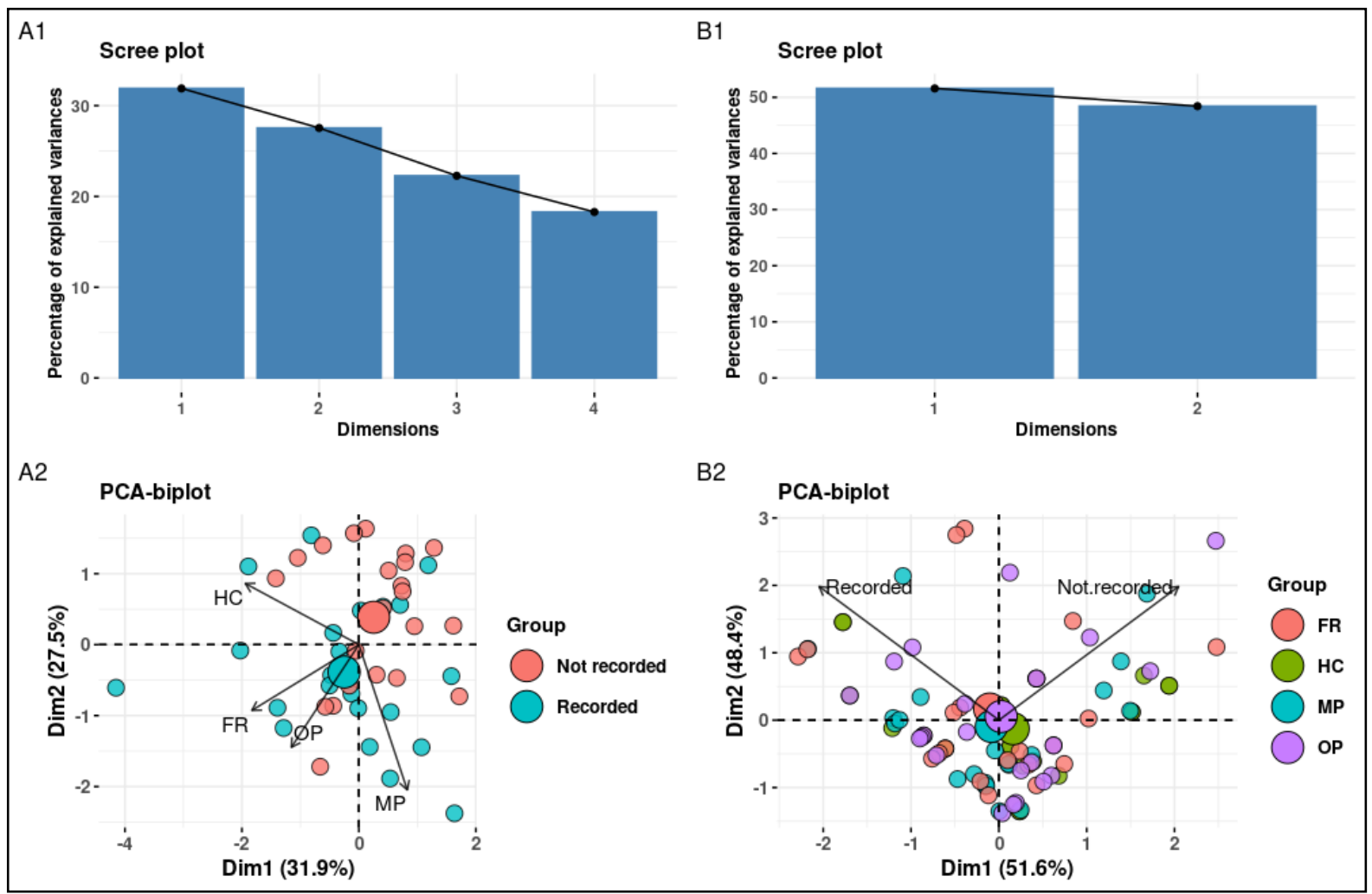

Figure 5. PCA biplot describing the data distributing and correlation between parameters [FR $=$ Forage, $\mathrm{HC}=$ Human consumption, MP = Medicinal use, $\mathrm{OP}=$ Other use $]$.

\section{Use value}

We recorded use values of 40 Elatostema species in our study. These values ranged from 0.18 to 1 (Appendix-2). In our study lower were the values, higher were the recorded usage and citations, for example, E. platyphyllum having UV 0.18 actually has the highest number of recorded usage and citation in our study and thus, a lower index, while on the other hand E. rostratum, E. monandrum, E. sp1, etc. having UV 1 actually have least number of recorded usage and citations in our study and higher index (Appendix-2).

\section{Discussion}

Loss of biodiversity is happening at an increasing rate across the globe especially in the forested areas near human habitations. The dependency of human beings on a few selected plant species and processed foods has also added to this destruction (Levin \& Wilson 1976; Padro-De-Santayana et al. 2005; Goodman 2015). While agricultural societies depend mostly on few domesticated species, the indigenous people depend on wild plants for their nutrition (Konsam et al. 2016a). Since, they do not have large land holdings and are unable to afford basic agricultural equipment, they incorporate number of unconventional food plants in their diet (Samant \& Dhar 1997; Padro-De-Santayana et al. 2005). Wild edible plants tend to be used as supplement or substitutes of food in times of dearth. Ethnobotanical studies play an important role in providing information about usage of wild plants and their morphology (Dogan et al. 2004). Elatostema is one such genus of interest with varied recorded usage by indigenous people in different parts of the world. Though leaf area does not show any relation to specific usage of species with in this genus, leaf size and morphology could be a target for selection of species in this genus for various ethnobotanical usage or even for their selection in bringing them for introduction to the mainstream food chain and also to develop proper agro-technological methods for cultivation. Moreover, variation in usage of Elatostema species shows its broad application.

\section{Ethnobotanical analyses for species diversity and usage}

Ethnobotanical studies provide a wide range of data on preparation methods, usage, medicinal values, toxicity and distribution of wild edible plants (Casas et al. 1996). It also shows how and why people selected specific plants for ethnobotanical usage and if there is a relation between the usage and availability of plants (Gaoue et al. 2017).

In our datasets we recorded 40 Elatostema species with ethnobotanical uses as food, medicinal, forage and others (material and ornamental). These uses were attributed to 30 indigenous communities across the world. Some 30 species recorded in our datasets were seen to have only a single usage, while 9 species were recorded to have two or more usage.

Among various indigenous communities, wild plants are an essential source of medicine to treat diseases and nearly $80-85 \%$ of population in emerging countries use conventional plants to treat various diseases and illness (Hamilton 2004; Dias et al. 2010; ljaz et al. 2020). 
The most frequent use of Elatostema species in our study is also as medicine followed by food, forage and material uses. Studies have shown that the antioxidant and cholinesterase inhibitory activities of E. papillosum leaves shows potential for treatment of Alzheimer's disease (Reza et al. 2018). We identified 20 Elatostema species (Table-2) used for medicinal purposes by various indigenous communities. Leaf was the most frequently used part for most of the medicinal preparations. Only in the cases of $E$. integrifolium, E. platyphyllum and E. monandrum the root juice was extracted and was used for treating fever, for inducing vomiting and for treating cuts and wounds respectively. Five species, $E$. sessile, $E$. platyphyllum, E. papillosum, E. paivaeanum and $E$. strigosum were used to treat gastrointestinal disorders and abdominal pain. Studies on plants to improve symptoms of Gastroesophageal reflux disease (GERD) conducted by Salehi et al. (2017) also shows that the antioxidant and antiinflammatory properties of many wild plants helps in ameliorating the effects of gastrointestinal disorders. Furthermore, it also helps in increasing gastric mucous and decreasing gastric acids which helps in alleviating pain caused by stomach disorders (Salehi et al. 2017).

We recorded 16 species which are used for human consumption. Again, leaves were the most used plant part. E. macrophyllum was used as a salt substitute by the people of Samawa community (Indonesia) and indigenous communities of Papua New Guinea as in these areas the salt or salt water was difficult to procure therefore, leaf ashes of $E$. macrophyllum was used. This usage is attributed only to these communities as the availability of this species is recorded only from those areas (Rugayah et al. 1989).

Though we found uses of Elatostema from various countries, the depth of usage and documentation of usage was found to be more in India. We recorded 15 indigenous communities that use Elatostema for various purposes and of these communities, 14 were recorded to reside in North East India. This region is endowed with rich resources of land, water, forests and minerals and is inhabited by ca. 130 indigenous communities (Sengupta 2003). The usage of Wild edible plants in these areas were recorded to be higher than central part of India where the species diversity was low and very few indigenous communities remain (Samant \& Dhar 1997). Even though the sources for collection of ethnobotanical data included literature survey from various areas and in different languages (Mandarin, Indonesian and Japanese etc.), there could be a disparity in the data due to the reason that we primarily used resources from Central National Herbarium (CAL) library and thereby, biasing the details of our study to India and to resources published in English language.

\section{Use-value (UV) within the recorded species}

Use-Value across recorded categories (Food, Medicinal, Forage and Others) and their citations showed that higher the usage and citations, lower the UV. So, in our case Use-values near 0 and under
1 had more recorded usage and citations while values over 1 had only one or two recorded usage and only one citation. E. platyphyllum $(U V=0.18)$ and $E$. sessile (UV=0.23) have the maximum number of recorded usage and citations and thus have the lowest UV. These two species were also recorded to be used by maximum number of indigenous communities across the world. Species with UV $>1$ were seen to have only one or two recorded usage and citations. The reason for few recoded usage and citations could be associated to the restricted geographical range and endemism, as species such as E. beccarii H.Schroet., E. paivaeanum Wedd. and $E$. strigosum Hassk. were recorded to have very limited native range. The geographic distinctiveness could have resulted in limited usage by the indigenous communities residing in those areas. $E$. beccarii was used only by Melpas since this plant species is only found in Papua New Guinea (Telban 1988). Likewise, E. paivaeanum and E. strigosum were used only by Chaggas and Batak Simalungans respectively as these tribes reside in the native ranges from where these species have been identified and recorded.

\section{Elatostema producing income for indigenous people}

Wild plants have become a major source of income for indigenous communities (Salam et al. 2012; Muthu \& Rimo 2018). These plants are sold in local markets at a very cheaper rate. Wild edible plants such as Diplazium sp., Piper pedicellatum C. DC. and Pilea sp. were observed to be sold in the market along with $E$. sessile and $E$. platyphyllum in Arunachal Pradesh (Personal Observation). These plants are directly plucked or chopped off from their natural habitats and are sold for INR 20 (\$0.27 USD) to INR 30 (\$0.41 USD) for 500 grams in the local markets (Pfoze et al. 2012; Nyitan \& Das 2018). The demand for these leafy vegetables especially Pilea and Elatostema species has increased in the local markets due to their availability all-round the year (Muthu \& Rimo 2018). Konsam et al. (2016a) noted the high value of $E$. lineolatum along with other leafy vegetables used by ethnic communities of Manipur, India. This was attributed to the traits such as unique taste, edible parts, high abundance, ease of collection, processing and high market value of these plants.

\section{Conclusion}

This study illustrates diversity of Elatostema species used for human consumption, medicine, forage and ornamental or material purposes by various indigenous communities in the world. The study disproves our hypothesis that the usage of an Elatostema species for a specific purpose (human consumption, medicine, fodder, others) is related to leaf size, i.e., bigger the size of the leaves, more the chances of it to be used as food or forage. Results from our analyses shows that there is no variation in leaf area with the type of use. The documented ethnobotanical records of Elatostema species along with our personal observations in the field provided in this study would help in elucidating the importance of this genus as one of the main leafy wild edible plant for human consumption and will further 
promote applied research in this group. Elatostema species can be further studied for their nutraceutical, anti-oxidant, medicinal and chemical properties and they can be brought under domestication and breeding in future. We further suggest comprehensive morphological and molecular studies of the related wild species with ethnobotanical uses for better identification of a promising candidate for domestication.

\section{Declarations}

List of abbreviations: WEP: Wild Edible plants, PCA: Principal Component Analysis, UV: Use-Value, NTFP: Non-Timber Forest Product, IPNI: International Plant Name Index

Ethics approval and consent to participate: Not applicable

Consent for publication: Not Applicable

Availability of data and material: The data was not deposited any public repositories.

Competing interests: The authors declare no competing interests.

Funding: This study has received partial funding from Botanical Survey of India, Kolkata.

Authors' contributions: AKU was involved in data collection, literature survey and identification of plants. RG supervised the work and gave important inputs for the study. PKM was involved in conducting all the statistical analyses. All the authors (AKU, RG \& PKM) contributed towards writing the manuscript.

\section{Acknowledgements}

The authors would like to thank Dr. A.A. Mao, Director, Botanical Survey of India for providing facilities and support. The authors are grateful to Dr. Kumar Avinash Bharti, Botanical Survey of India, for his valuable suggestions in interpretation of data. Thanks are due to Prof. A.K. Pandey for providing valuable suggestions for improvement of the manuscript. The authorities of the forest department of different states of North East India are also acknowledged for their help and cooperation.

\section{Literature cited}

Albuquerque UP, Hanazaki N. 2009. Commentary: Five problems in current ethnobotanical research and some suggestions for strengthening them. Human Ecology 37:653-661.

Angami A, Gajurel PR, Rethy P, Singh B, Kalita SK. 2006. Status and potential of wild edible plants of Arunachal Pradesh. Indian Journal of Traditional Knowledge 5(4):541-550.

Anonymous. 1952. The Wealth of India- A dictionary of India Raw Materials and Industrial Products. Vol. III: D-E. p. 142. Council of Scientific and Industrial Research, New Delhi

Arora RK \& Pandey A. 1996. Wild edible plants of India, Diversity, Conservation and Use. National Bureau of Plant Genetic Resources (NBPGR), ICAR, New Delhi.

Awika JM. 2011. Major Cereal Grains Production and Use around the World. In: Awika JM, Piironen V, Bean $S$ (Eds.) Advances in Cereal Science:
Implication to food processing and Health promotion. ACS symposium series. 1089:1-13. doi: 10.1021/bk2011-1089.ch001

Badola HK, Pradhan BK. 2013. Plants used in healthcare practices by Limboo tribe in South-West of Khangchendzonga Biosphere Reserve, Sikkkim, India. Indian Journal of Traditional Knowledge. 12(3): 355-369.

Bharti KA, Sharma BL. 2012. Plants used as Ethnoveterinary Medicines in Sikkim Himalayas. Ethnobotany Research \& Applications 10:339-356.

Bhatt M, Chanda SV. 2003. Prediction of Leaf Area in Phaseolus vulgaris by Non-Destructive Method. Bulgaria Journal of Plant Physiology 29:96-100.

BHL. 2020. Biodiversity Heritage Library (https://www.biodiversitylibrary.org/) (Retrieved 20 August 2020).

Borah D, Tangjang S, Das AP, Upadhya A, Mipun P. 2020. Assessment of Non-Timber Forest products (NTFPs) in Behali Reserve Forest, Assam, Northeast India. Ethnobotany Research \& Applications 19(43):1-15.

Borthakur SK. 1976. Less known medicinal uses of Plants among the tribes of Karbi-Anglong (Mikir Hills), Assam. The Bulletin of Botanical Survey of India 18(1-4):166-171.

Casas A, Vázquez MDC, Viveros JL, Caballero J. 1996. Plant Management among the Nahua and the Mixtec in the Balsas River Basin, Mexico: An Ethnobotanical approach to the study of Plant Domestication. Human Ecology 24(4):455-478.

Dias DA, Urban S, \& Roessner U. 2012. A historical overview of natural products in drug discovery. Metabolites 2(2):303-336. https ://doi.org/10.3390/metab 02020303.

Dogan Y, Balasar S, Ay G, Mert HH. 2004. The use of Wild edible plants in Western and Central Anatolia (Turkey). Economic Botany 58(4):648-690.

Fathurrahman F, Nursanto J, Madjid A \& Ramadanil R. 2016. Ethnobotanical study of "Kaili Inde" tribe in Central Sulawesi Indonesia. Emirates Journal of Food and Agriculture 28(5):337-347. doi:10.9755/ejfa.2015-06-463

Gaoue OG, Coe MA, Bond M, Hart G, Seyler B.C., Mcmillen H. 2017. Theories and major hypotheses in Ethnobotany. Economic Botany 20(10):1-19.

Gardner RO. 2010. Plant names of the Kalam (Upper Kaironk Valley, Schrader range, Papua New Guinea). Records of Auckland Museum 47:5-50.

GBIF Secretariat. 2020. GBIF Backbone. Taxonomy. https://doi.org/10.15468/39omei Accessed via https://www.gbif.org/species/5284517 [Accessed on 18 August 2020] https://www.gbif.org/species/search?q=Elatostema \&rank=SPECIES\&status $=$ ACCEPTED 
Geng Y, Hu G, Ranjitkar S, Wang Y, Bu D, Pei S, Ou X, Lu Y, Ma X, Xu J. 2017. Prioritizing fodder species based on traditional knowledge: a case study of Mithun (Bos frontalis) in Dulongjiang area, Yunnan Province, Southwest China. Journal of Ethnobiology and Ethnomedicine 13(24):1-15. doi: 10.1186/s13002-017-0153-Z

Gewali MB. 2008. Aspects of Traditional Medicine in Nepal. Institute of natural Medicine, University of Tokoyama. Pp. 1-175.

Ghorbani A, Langerberger G, Sauerborn J. 2012. A comparison of the wild food plant use knowledge of ethnic minorities in Naban River Watershed National Nature Reserve, Yunnan, S.W. China. Journal of Ethnobiology and Ethnomedicine 8(17):1-9.

Goodman DM. 2015. The McDonaldization of psychotherapy: Processed foods, processed therapies, and economic class. Theory and Psychology 1-19. doi: 10.1177/0959354315619708

Hadiah JT, Conn BJ. 2009. Usefulness of morphological characters for infrageneric classification of Elatostema (Urticaceae). Blumea 54:181-191. doi: 10.3767/000651909X476139

Hadiah JT, Quinn CJ, Conn BJ. 2003. Phylogeny of Elatostema (Urticaceae) using chloroplast DNA data. Telopea 10(1):235-246.

Hamilton AC. 2004. Medicinal plants, conservation, and livelihoods. Biodiversity Conservation, 13:14771517.

Harada K. 2004. Dependency of local people on the forests of Gunung Halimun National Park, West Java, Indonesia. Tropics 13(3):161-185.

Hays TE. 1980. Uses of Wild Plants in Ndumba, Eastern Highlands Province. Science in New Guinea 7(3):118-131.

Hemp A. 1999. An Ethnobotanical study on Mt. Kilimanjaro. Ecotropica 5:147-165.

Heywood VH. 2011. Ethnopharmacology, food production, nutrition and biodiversity conservation: towards a sustainable future for indigenous peoples. Journal of Ethnopharmacology 137(1):1-15.

Hui PK. 2019. Survey and Validation Studies on Local Food and Medicinal Bioresources of Kameng and Tawang Districts of Arunachal Pradesh for Supplementary Rural Livelihood Security. Project Report. Integrated Eco-development Research Programme, G.B. Pant National Institute of Himalayan Environment and Sustainable development. Pp. 1-19.

Ibo LK, Arimukti SD. 2019. Ethnobotanical study of Batak Toba sub-ethnic community in Martoba Village, Samosir District, North Sumatra. Prosiding Seminar Nasional Masyarakat Biodiversitas Indonesia 5(1):234-241.

ljaz S, Perveen A, Ashraf S, Bibi A, Dogan Y. 2020. Indigenous wild plants and fungi traditionally used in folk medicine and functional food in District Neelum Azad Kashmir. Environment, Development and Sustainability doi: https://doi.org/10.1007/s10668020-00966-w

IPNI. 2020. International Plant Names Index. Published on the Internet http://www.ipni.org, The Royal Botanic Gardens, Kew, Harvard University Herbaria \& Libraries and Australian National Botanic Gardens. [Retrieved 07 August 2020].

Jain SK, Dam N. 1979. Some Ethnobotanical Notes from North Eastern India. Economic Botany 33(1):52-56.

Jaiswal V. 2010. Culture and ethnobotany of Jaintia tribal community of Meghalaya, Northeast India- A mini review. Indian Journal of Traditional Knowledge 9(1):38-44.

Jha KK. 2015. Non-timber Forest Products, Their Vulnerability and Conservation in a designated UNESCO Heritage Site of Arunanchal Pradesh, India. Notulae Scientia Biologicae 7(4):444-455. doi: 10.15835/nsb.7.4.9701.

JSTOR. 2020. Published on the Internet (https://www.jstor.org/)_(Retrieved 20 August 2020).

Kala CP. 2005. Ethnomedicinal botany of the Apatani in the Eastern Himalayan region of India. Journal of Ethnobiology and Ethnomedicine 1(11):18. doi: 0.1186/1746-4269-1-11.

Kar A, Bora D, Borthakur SK, Goswami NK, Saharia D. 2013. Wild edible plant resources used by Mizos of Mizoram, India. Kathmandu University Journal of Science, Engineering and Technology 9(1):106-126.

Kar A, Borthakur SK. 2007. Wild vegetables sold in local markets of Karbi Anglong, Assam. Indian Journal of Traditional Knowledge 6(1):169-172.

Koch M, Kehop DA, Kinminja B, Sabak M, Wavimbukie G, Barrows KM, Matainaho TK, Barrows LR, Rai PP. 2015. An ethnobotanical survey of medicinal plants used in East Sepik province of Papua New Guinea. Journal of Ethnobiology and Ethnomedicine 11(79):1-26. doi: 10.1186/s13002015-0065-8.

Konsam S, Thongam B, Handique AK. 2016a. Assessment of wild leafy vegetables traditionally consumed by the ethnic communities of Manipur, northeast India. Journal of Ethnobiology and Ethnomedicine 12(9):1-15. doi 10.1186/s13002-0160080-4

Konsam SC, Devi KT, Salam JS, Singh PK. 2016b. Biochemical Constituents and Nutritive Evaluation of Some Less Known Wild Edible Plants from Senapati District, Manipur, India. Notulae Scientia Biologicae 8(3):370-372. doi: 10.15835/nsb.8.3.9871.

Kumar SJU, Chaitanya KMJ, Semotiuk AJ, Krishna V. 2019. Indigenous knowledge of medicinal plants used by ethnic communities of South India. Ethnobotany Research \& Applications 18(4):1-112. 
Lalmuanpuii R, Laha RL, Lalremruata PC. 2018. Diversity assessment of wild edible vegetables in Aizawl District, Mizoram, North East India. International Journal of Basic and Applied Research 8(9):45-62.

Lescure JP, Pinton P, Emperaire L. 1994. People and forest products in Central Amazonia: the multidisciplinary approach of extractivism. MAB digest 18:58-87.

Levin DA, Wilson AC. 1976. Rates of evolution in seed plants: Net increase in diversity of chromosome numbers and species numbers through time. Proceedings of the National Academy of Sciences 73:2086-2090.

Li S, Zhang Y, Guo Y, Yang L, Wang Y. 2020. Monpa, memory, and change: an ethnobotanical study of plant use in Mêdog County, South-east Tibet, China. Journal of Ethnobiology and Ethnomedicine 16(5):1-26. doi: 10.1186/s13002020-0355-7

Lou B, Liu B, Zhang H, Zhang H, Li X, Ma L, Wang Y, Bai Y, Zhang X, Li J, Yang J, Long C. 2019. Wild edible plants collected by Hani from terraced rice paddy agroecosystem in Honghe Prefecture, Yunnan, China. Journal of Ethnobiology and Ethnomedicine 15(56):1-22. doi: 10.1186/s13002019-0336-x

Maikhuri RK, Rao KS, Saxena KG. 2004. Bioprospecting of wild edibles for rural development in the central Himalayan mountain of India. Mountain Research and Development 24(2):110-113.

Manandhar NP. 1993. Ethnobotanical note on folklore remedies of Baglung district, Nepal. Contributions to Nepalese Studies 20(2):183-195.

Mariani R, Suganda AG, Sukandar EY. 2016. Drugdrug interactions between riseofulvinand a new prenylated chalcone from Elatostema parasiticum and its antibacterial activity nortriptylineat binding sites of bovine serum albumin. Pharmacology Online 1:1-6.

Miller AJ, Novy A, Glover J, Kellogg EA, Maul JE, Raven P, Jackson W. 2015. Expanding the role of Botanical gardens in the future of food. Nature Plants 1:15078. doi: 10.1038/NPLANTS.2015.78

Murtem G, Chaudhry P. 2016a. An ethnobotanical note on wild edible plants of Upper Eastern Himalaya, India. Brazilian Journal of Biological Sciences 3(5): 63-81. doi: 10.21472/bjbs.030506

Murtem G, Chaudhry P. 2016b. An Ethnobotanical Study of Medicinal Plants Used by the Tribes in Upper Subansiri District of Arunachal Pradesh, India. American Journal of Ethnomedicine 3(3):35-49.

Muthu J, Rimo Y. 2018. An enumeration on some of the commercialized Ethno-Vegetables Plants of Arunachal Pradesh: A preliminary study. Bulletin of Arunachal Forest Research 33(1): 2-58.
Nyitan J, Das AK. 2018. Documentation and phytochemical screening of wild vegetables from Siang District of Arunachal Pradesh, India. Pleione 12(2):231-241.

doi:

10.26679/Pleione.12.2.2018.231-241

Ohtsuka R, Suzuki T, Morita M. 1987. Sodium-rich Tree ash as a native salt source in Lowland Papua. Economic Botany 41(1):55-59.

Pal GD. 1984. Observations on Ethnobotany of tribals of Subansiri, Arunachal Pradesh. The Bulletin of Botanical Survey of India 26(1\&2):26-37.

Pardo-De-Santayana M, Tardío J, Morales R. 2005. The gathering and consumption of wild edible plants in the Campoo (Cantabria, Spain). International Journal of Food Sciences and Nutrition 56(7):529542. doi:10.1080/09637480500490731

Paulsamy S, Vijayakumar KK, Murugesan M, Padmavathy S, Senthilkumar P. 2007. Ecological status of medicinal and other economically important plants in the Shola understories of Nilgiris, the Western Ghats. Natural Product Radiance 6(1):5561.

Pfoze NL, Kumar Y, Sheikh N, Myrboh B. 2012. Assessment of local dependency on selected Wild Edible Plants and Fruits from Senapati district, Manipur, Northeast India. Ethnobotany Research \& Applications 10:357-367.

Phillips OL, Gentry AH. 1993. The useful plants of Tambopata, Peru: I. Statistical hypothesis tests with a new quantitative technique. Economic Botany 47:15-32.

Phumthum M, Balslev H. 2018. Thai Ethnomedicinal Plants Used for Diabetes Treatment. OBM Integrative and Complementary Medicine 3(3):1-25. doi: 10.21926/obm.icm. 1803020

Pieroni A, Giusti ME, Quave CL. 2011. Cross-cultural ethnobiology in the Western Balkans: Medical ethnobotany and ethnozoology among Albanians and Serbs in the Pešter Plateau, Sandžak, South Western Serbia. Human Ecology 39:333-349. doi :10.1007/s10745-011-9401-3

Plants for future. 2020. Published on the Internet; (https://pfaf.org/) (accessed 20th August 2020).

Powell B, Ouarghidi A, Johns T, Tattou MI, Eyzaguirre P. 2014. Wild leafy vegetable use and knowledge across multiple sites in Morocco: a case study for transmission of local knowledge? Journal of Ethnobiology and Ethnomedicine 10(34):1-11.

POWO. 2020. Plants of the World Online. Facilitated by the Royal Botanic Gardens, Kew. Published on the Internet; http://www.plantsoftheworldonline.org/ Retrieved 20/08/2020.

Pradhan BK, Badola HK. 2008. Ethnomedicinal plant use by Lepcha tribe of Dzongu valley, bordering Khangchendzonga Biosphere Reserve in North 
Sikkim, India. Journal of Ethnobiology and Ethnomedicine 4:22. doi: 10.1186/1746-4269-4-22

Pubmed. 2020. Published on the Internet (https://pubmed.ncbi.nlm.nih.gov/) (Retrieved 20 August 2020).

Purba EC, Nisyawati, Silalahi M. 2016. The ethnomedicine of the Batak Karo people of Merdeka sub-district, North Sumatra, Indonesia. International Journal of Biological Research. 4(2):181-189 doi:10.14419/ijbr.v4i2.6493

Qi L, Friis I, Wilmot-Dear MC. 2003. Elatostema J.R. Forster \& G. Forster, Char. Gen. PI. 53. 1775, nom. cons. In: Wu, Z. \& P.H. Raven (Eds.), Flora of China. Vol. 5: 76-189. Science Press, Beijing and Missouri Botanical Garden Press, St. Louis, Missouri.

Rahayu M, Rustiami H. 2017. Ethnobotani Masyarakat Samawa Pulau Sumbawa. Scripta Biologica 4(4):235-245. doi: 10.20884/1.SB.2017.4.4.605

Rahman MA, Uddin SB, Wilcock CC. 2007. Medicinal plants used by Chakma tribe in Hill tracts districts of Bangladesh. Indian Journal of Traditional Knowledge 6(3):508-517.

Reza ASMA, Nasrin MS, Alam AHMK. 2018. Phytochemicals, Antioxidants, and Cholinesterase Inhibitory Profiles of Elatostema papillosum Leaves: An Alternative Approach for Management of Alzheimer's Disease. Journal of Neurology \& Neuromedicine 3(5):19-22.

Rinya M, Bhagwati P, Kar A. 2020. Traditional knowledge of herbal medicines practiced by Apatani tribe in the Ziro Valley of Arunachal Pradesh, India. Pleione. 14(1): 129-135. doi: 10.26679/Pleione.14.1.2020.129-135

Ronald K, Lodhi MS, Singha R, Kumari S, Kanwal KS, Arya SC. 2019. Wild edible plants used by the ethnic communities of Shi Yomi District of Arunachal Pradesh, India. Pleione 13(2):247-257. doi: 10.26679/Pleione.13.2.2019.247-257

Rossato SC, Leitão-Filho HFD, Begossi A. 1999. Ethnobotany of Caiçaras of the Atlantic forest coast (Brazil). Economic Botany 53(4):387-395.

RStudio Team. 2020. RStudio: Integrated Development for R. RStudio, PBC, Boston, MA URL http://www.rstudio.com/.

Rugayah DS, Jarwaningsih, Widjaja EA. 1989. South-East Asian Spices: Present state and Future prospects as exemplified by Indonesian Cooking. Pp.154-163.In: Siemonsma, J.S. \& N.W. Soetjipto (Eds.). Proceedings of the First PROSEA International Symposium, Jakarta, Indonesia. $337 \mathrm{pp}$.

Saha D, Sundriyal RC. 2013. Perspectives of Tribal Communities on NTFP Resource Use in a Global
Hotspot: Implications for Adaptive Management. Journal of Natural Science Research 3(4):125-169.

Salam S, Jamir NS, Singh PK. 2012. Wild leafy vegetables sold in local markets of Ukhrul District of Manipur, India. Pleione 6(2):298-303.

Salehi M, Karegar-Borzi H, Karimi M, Rahimi R. 2017. Medicinal Plants for Management of Gastroesophageal Reflux Disease: A Review of Animal and Human Studies. The Journal of Alternative and Complementary Medicine 23(2):8295. doi: 10.1089/acm.2016.0233

Samant SS, Dhar U. 1997. Diversity, endemism and economic potential of wild edible plants of Indian Himalaya. International Journal of Sustainable Development and World Ecology 4(3):179-191.

Santos TC, Gomes TM, Pinto BAS, Camara AL, Paes AMA. 2018. Naturally occurring Acetylcholinesterase Inhibitors and their potential use for Alzheimer's disease therapy. Frontiers in Pharmacology. 9:1-14. doi: 10.3389/fphar.2018.01192

Sengupta S. 2003. Tribes of North East IndiaBiological and Cultural Perspectives. Gyan Publishing House, New Delhi. Pp. 1-20.

Shrestha D. 2013. Indigenous vegetables of Nepal for biodiversity and food security. International Journal of Biodiversity and Conservation 5(3):98108. doi:10.5897//JBC11.124

Shrestha I. 2015. Impact on Medicinal plants by earthquake and landslide in Langtang Village Development Committee (VDC), Nepal. GIS Nepal 13:13-17.

Sigdel SR, Rokaya MB, Timsina B. 2013. Plant inventory and ethnobotanical study of Khimti hydropower project, Central Nepal. Scientific World 11(11):105-112.

Silalahi M, Nisyawati, Walujo EB, Mustaqim W. 2018. Ethnomedicine of medicinal plants by Batak Phakpak Sub-ethnic in The Surung Mersada Village, Phakpak Bharat District, North Sumatera. Jurnal ILMU DASAR 19(2):77-92.

Silalahi M, Supriatna J, Walujo EB, Nisyawati. 2015. Local knowledge of medicinal plants in sub-ethnic Batak Simalungun of North Sumatra, Indonesia. Biodiversitas 16(1):44-54.

Singh AB, Teron R. 2015. Diversity of wild edible plants used by the Angami-Nagas in Kohima District of Nagaland, India. Pleione 9(2):311-324.

Srivastava RC, Adi Community. 2009. Traditional knowledge of Adi tribe of Arunachal Pradesh on Plants. Indian Journal of Traditional Knowledge 8(2):146-153.

Srivastava RC, Nyishi Community. 2010. Traditional knowledge of Nyishi (Daffla) tribe of Arunachal Pradesh. Indian Journal of Traditional Knowledge 9(1):26-37. 
Stefan M, Bîrsă ML. 2019. Flavonoids - An amazing group of compounds with potent antimicrobial properties. Memoirs of the Scientific Sections of the Romanian Academy 42:111-139.

Sundriyal M, Sundriyal RC, Sharma E, Purohit AN. 1998. Wild edible and other useful plants from Sikkim Himalaya, India. Oecologia Montana 7:43-54.

Tag H, Jeri L, Mingki T, Tsering J, Das AK. 2012. Higher Plant Diversity in Pakke Wildlife Sanctuary and Tiger Reserve in East Kameng District of Arunachal Pradesh: Checklist - I. Pleione 6(1):149162.

Tedder J, Tedder M. 1979. Magic and Medicine in Solomon Islands. Journal Anthropological Society of South Australia 17(5):26-32.

Telban B. 1988. The role of medical ethnobotany in Ethnomedicine: A New Guinea example. Journal of Ethnobiology 8(2):149-169.

Thiers B. 2016. Index herbariorum: a global directory of public herbaria and associated staff. New York Botanical Garden's Virtual Herbarium. http://sweetgum.nybg.org/ih

Tseng YH, Monro AK, Wei YG, Hu JM. 2019. Molecular phylogeny and morphology of Elatostema s.l. (Urticaceae): Implications for inter- and infra generic classifications. Molecular Phylogenetics and Evolution 132:251-264. doi: 10.1016/j.ympev.2018.11.016.

Tsering J, Gogoi BJ, Hui PK, Tam N, Tag H. 2017. Ethnobotanical appraisal on wild edible plants used by the Monpa community of Arunachal Pradesh. Indian Journal of Traditional Knowledge 16(4):626637.

Tshering D, Das S, Tsering J, Hui PK, Tag H. 2018. Rapid ethnobotanical appraisal on Bugun, Sartang and Monpa communities of West Kameng and Tawang sectors of Arunachal Himalayan Region, India. Pleione 12(2):283-297.

Vilash V, Suja SR, Latha PG, Shine VJ, Rajasekharan S. 2016. Chronic oral toxicity studies of crude ethanolic extract and ethanolic fraction of Pellionia heyneana Wedd. leaf in wistar rats. International Journal of Pharmacy and Pharmaceutical Sciences 8(8):306-312.

Wei YG, Monro AK, Wang WT. 2011. Additions to the Flora of China: seven new species of Elatostema (Urticaceae) from the karst landscapes of Guangxi and Yunnan. Phytotaxa 29:1-27.

Wiley SP, Eguchi A, Kinoshita I. 2019. Psychological ownership of Ethnobotany for strengthening SocialEcological Systems. Journal of Architecture and Planning, Japan 84(759):1135-1144.

Wu ZY, Monro AK, Milne RI, Wang H, Yi TS, Liu J, Li DZ. 2013. Molecular phylogeny of the nettle family (Urticaceae) inferred from multiple loci of three genomes and extensive generic sampling. Molecular Phylogenetics and Evolution 69:814-827. doi: 10.1016/j.ympev.2013.06.022

Yakang B, Gajurel PR, Potsangbam S, Bhuyan LR. 2013. Account of common and traditional non-timber forest products used by Apatani tribe of Arunachal Pradesh, India. Pleione 7(2):514-529.

Yumnam JY, Bhuyan SI, Khan ML, Tripathi OP. 2011. Agro-diversity of East Siang-Arunachal Pradesh, Eastern Himalaya. Asian Journal of Agricultural Sciences.3(4):317-326.

Yumnam JY, Bhuyan SI, Tripathi OP, Khan ML. 2011. Study on ethno-medicinal plants used by Adi tribe of East Siang District, Arunachal Pradesh. Journal of Economic and Taxonomic Botany 35(2):369-377.

Zenderland J, Hart R, Bussmann RW, Paniagua Zambrana NY, Sikharulidze S, Kikvidze Z, Kikodze $D$, Tchelidze D, Khutsishvili M, Batsatsashvili K. 2019. The use of "Use Value: Quantifying Importance in Ethnobotany. Economic Botany 73(1):1-11. doi: 10.1007/s12231-019- 09480-1 


\section{Appendices}

Appendix 1. Leaf area of Elatostema species recorded in the study. Taxa identified up to the rank of species are taken for leaf area measurements, 13 taxa that are not identified up to the rank of species are not used for leaf area measurements.

\begin{tabular}{|l|l|}
\hline Species & Leaf area $\mathbf{( c m}$ ) \\
\hline Elatostema dissectum Wedd. & $70 \mathrm{~cm}^{2}$ \\
\hline Elatostema cuneatum Wight & $4.06 \mathrm{~cm}^{2}$ \\
\hline Elatostema laetevirens Makino & $11.25 \mathrm{~cm}^{2}$ \\
\hline Elatostema sessile J.R. Forst. \& G. Forst. & $70 \mathrm{~cm}^{2}$ \\
\hline Elatostema integrifolium (D. Don) Wedd. & $48 \mathrm{~cm}^{2}$ \\
\hline Elatostema lineolatum Wight & $43.75 \mathrm{~cm}^{2}$ \\
\hline Elatostema beccarii H. Schroet. & $10.31 \mathrm{~cm}^{2}$ \\
\hline Elatostema platyphyllum Wedd. & $182.75 \mathrm{~cm}^{2}$ \\
\hline Elatostema cuneiforme W.T. Wang & $86.25 \mathrm{~cm}^{2}$ \\
\hline Elatostema nasutum Hook. f. & $26 \mathrm{~cm}^{2}$ \\
\hline Elatostema paivaeanum Wedd. & $12.68 \mathrm{~cm}^{2}$ \\
\hline Elatostema umbellatum (Siebold \& Zucc.) Blume & $10 \mathrm{~cm}^{2}$ \\
\hline Elatostema papillosum Wedd. & $34.12 \mathrm{~cm}^{2}$ \\
\hline Elatostema strigosum Hassk. & $32.24 \mathrm{~cm}^{2}$ \\
\hline Elatostema acuminatum (Poir.)B rongn. & $26.25 \mathrm{~cm}^{2}$ \\
\hline Elatostema longipes W.T. Wang & $35 \mathrm{~cm}^{2}$ \\
\hline Elatostema involucratum Franch. \& Sav. & $8.8 \mathrm{~cm}^{2}$ \\
\hline Elatostema parasiticum Blume ex H. Schroet. & $108.75 \mathrm{~cm}^{2}$ \\
\hline Elatostema ficoides Wedd. & $105 \mathrm{~cm}^{2}$ \\
\hline Elatostema parvum (Blume) Blume ex Miq. & $7.12 \mathrm{~cm}^{2}$ \\
\hline Elatostema hookerianum Wedd. & $11.25 \mathrm{~cm}^{2}$ \\
\hline Elatostema laevissimum W.T. Wang & $37.18 \mathrm{~cm}^{2}$ \\
\hline Elatostema macrophyllum Brongn. & $139.46 \mathrm{~cm}^{2}$ \\
\hline Elatostema rupestre (Buch.-Ham. ex D. Don) Wedd. & $27.5 \mathrm{~cm}^{2}$ \\
\hline Elatostema monandrum (Buch.-Ham. ex D. Don) H.Hara & $4.37 \mathrm{~cm}^{2}$ \\
\hline Elatostema rostratum (ReinW. ex Blume) Hassk. & $103.61 \mathrm{~cm}^{2}$ \\
\hline Elatostema heyneanum (Wedd.) Hallier f. & $30 \mathrm{~cm}^{2}$ \\
\hline
\end{tabular}


Appendix 2. Use Value of Elatostema species recorded in the study

\begin{tabular}{|c|c|}
\hline Species & Use-Value \\
\hline Elatostema dissectum Wedd. & 0.33 \\
\hline Elatostema cuneatum Wight & 0.5 \\
\hline Elatostema laetevirens Makino & 0.5 \\
\hline Elatostema sessile J.R. Forst. \& G. Forst. & 0.23 \\
\hline Elatostema integrifolium (D. Don) Wedd. & 1 \\
\hline Elatostema lineolatum Wight & 0.4 \\
\hline Elatostema beccarii H. Schroet. & 1 \\
\hline Elatostema platyphyllum Wedd. & 0.18 \\
\hline Elatostema cuneiforme W.T. Wang & 2 \\
\hline Elatostema nasutum Hook. f. & 1.5 \\
\hline Elatostema paivaeanum Wedd. & 1 \\
\hline Elatostema umbellatum (Siebold \& Zucc.) Blume & 1 \\
\hline Elatostema papillosum Wedd. & 1 \\
\hline Elatostema strigosum Hassk. & 0.5 \\
\hline Elatostema acuminatum (Poir.) Brongn. & 1 \\
\hline Elatostema longipes W.T. Wang & 1 \\
\hline Elatostema involucratum Franch. \& Sav. & 1 \\
\hline Elatostema parasiticum Blume ex H. Schroet. & 0.5 \\
\hline Elatostema ficoides Wedd. & 1 \\
\hline Elatostema parvum (Blume) Blume ex Miq. & 1 \\
\hline Elatostema hookerianum Wedd. & 1 \\
\hline Elatostema laevissimum W.T. Wang & 1 \\
\hline Elatostema macrophyllum Brongn. & 0.66 \\
\hline Elatostema rupestre (Buch.-Ham. ex D. Don)Wedd. & 1 \\
\hline Elatostema monandrum (Buch.-Ham. ex D. Don) H. Hara & 1 \\
\hline Elatostema rostratum (Reinw. ex Blume) Hassk. & 1 \\
\hline Elatostema heyneanum (Wedd.) Hallier f. & 0.5 \\
\hline Elatostema sp 1. & 1 \\
\hline Elatostema sp 2. & 1 \\
\hline Elatostema sp 3. & 1 \\
\hline Elatostema sp 4. & 1 \\
\hline Elatostema sp 5. & 1 \\
\hline Elatostema sp 6. & 2 \\
\hline Elatostema sp 7. & 3 \\
\hline Elatostema sp 8. & 1 \\
\hline Elatostema sp 9. & 1 \\
\hline Elatostema sp 10. & 1 \\
\hline Elatostema sp 11. & 1 \\
\hline Elatostema sp 12. & 2 \\
\hline Elatostema sp 13. & 1 \\
\hline
\end{tabular}

